\title{
A Database of Narrow-Band Parameters for Fuels Commonly Encountered in Fire Applications
}

\author{
J. L. Consalvi ${ }^{\mathrm{a},{ }^{*}}$ and F. Liu ${ }^{\mathrm{b}}$ \\ ${ }^{a}$ Aix-Marseille University, IUSTI/ UMR CNRS 7343, 5 rue E. Fermi, 13453 Marseille Cedex 13, France. \\ ${ }^{\text {b }}$ Measurement Science and Standards, National Research Council of Canada, 1200 Montreal Road, Ottawa, \\ Ontario, Canada K1A 0R6 \\ * Corresponding author: Tel.: +33 491-106-831; Fax: +33-491-106-969. \\ E-mail address: jean-louis.consalvi@univ-amu.fr
}

\begin{abstract}
The objective of this paper is to derive narrow band (NB) parameters for the Malkmus statistical narrow band model from the medium resolution transmission measurements carried out at NIST (Wakatsuki et al., Proc. Combust. Inst. 31 (2007) 2573-2580) up to about $1000 \mathrm{~K}$ for nine fuels commonly encountered in fire applications, namely methane, methanol, ethane, ethylene, propane, propylene, heptane, methyl methacrylate and toluene. These NB parameters can be used to generate a database of NB k-distributions of these fuels in order to apply the k-distribution methods to model their contributions to radiative heat transfer. The accuracy of this NB database, the EM2C NB database for methane (Perrin and Soufiani, JQSRT 103 (2007) 3-13), and HITRAN 2012 for methane, methanol, ethane, and ethylene is assessed by comparison with the measured medium resolution transmissivities and spectrallyintegrated radiative properties. Results show that the EM2C database is the most accurate for methane over the entire range of the temperature and the fuel partial pressure path length considered. The line parameters in HITRAN 2012 are not available for some important bands
\end{abstract}


of methanol, ethane and ethylene and this spectroscopic database cannot be used with confidence above $600 \mathrm{~K}$. The Malkmus narrow band database derived in the present study is found to reproduce spectrally-integrated radiative properties with error less than $20 \%$, which suggests that it can be used for engineering applications. Finally, applications to radiation calculations along lines of sight with methane concentrations and temperatures representative of pool fires show that both the present Malkmus narrow band and the NIST databases can predict accurately the radiative intensity for small scale pool fires whereas discrepancies of the order of $30 \%$ are expected in the case of methane pool fires with a heat release rate of 176 $\mathrm{kW}$.

Key words: Fuels, Medium Resolution Transmission Measurements, Malkmus Model, Narrow Band Database, Narrow Band k-Distributions, Spectroscopic Database.

\section{Nomenclature}

$F \quad$ fraction of $\Delta \eta_{e}$ which belongs to $\Delta \eta[-]$

$f \quad$ k-distribution function [cm]

$g \quad$ cumulative k-distribution function [-]

$I_{b} \quad$ blackbody intensity (Planck function) $\left[\mathrm{W} / \mathrm{cm}^{2} / \mathrm{sr}\right]$

$k \quad$ absorption coefficient variable $\left[\mathrm{cm}^{-1}\right]$

$l \quad$ path length $[\mathrm{cm}]$

$p \quad$ pressure $[\mathrm{atm}]$

$R \quad$ ratio of the maximum to minimum value of the line intensity [-] 
$S \quad$ line intensity $\left[\mathrm{cm}^{-1} / \mathrm{mol} . \mathrm{cm}^{-2}\right]$

$S_{m} \quad$ maximum value of the line intensity $\left[\mathrm{cm}^{-1} / \mathrm{mol} . \mathrm{cm}^{-2}\right]$

$T \quad$ temperature $[\mathrm{K}]$

$x_{i} \quad$ mole fraction of $i^{\text {th }}$ species [-]

\section{Greek}

$\alpha \quad$ absorptance $\left[\mathrm{cm}^{-1}\right]$

$\beta_{M} \quad$ Average line width to line spacing ratio [-]

$\beta_{M S} \quad$ self broadening parameter in the Malkmus model [-]

$\beta_{M A}$ air broadening parameter in the Malkmus model [-]

$\Delta \eta \quad$ resolution of the NB database $\left[\mathrm{cm}^{-1}\right]$

$\Delta \eta_{e} \quad$ experimental resolution $\left[\mathrm{cm}^{-1}\right]$

$\varepsilon \quad$ emissivity [-]

$\kappa_{\eta} \quad$ spectral absorption coefficient $\left[\mathrm{cm}^{-1}\right]$

$\bar{\kappa}_{M} \quad$ Pressure based absorption coefficient $\left[\mathrm{atm}^{-1} \cdot \mathrm{cm}^{-1}\right]$

$\bar{\kappa}_{e} \quad$ pressure-based absorption coefficient deduced from $\bar{\tau}_{e}\left[\mathrm{~atm}^{-1} \cdot \mathrm{cm}^{-1}\right]$

$\eta \quad$ wavenumber $\left[\mathrm{cm}^{-1}\right]$

$\bar{\tau}_{e} \quad$ measured transmissivity at medium resolution of $1 \mathrm{~cm}^{-1}[-]$ 
$\bar{\tau}_{\text {ext }}$ extrapolated transmissivity at medium resolution of $1 \mathrm{~cm}^{-1}[-]$

$\bar{\tau}_{\Delta \eta} \quad$ reduced transmissivity with the NB spectral resolution of $25 \mathrm{~cm}^{-1}[-]$

$\sigma_{\mathrm{e}} \quad$ relative error on the measured transmissivity [-]

$\sigma_{\Delta \eta} \quad$ relative error on the reduced transmissivity [-]

\section{Subscript}

e experimental data

ext extrapolated

$\eta \quad$ wavenumber

\section{Abbreviations}

FS Full spectrum

MMA Methyl methacrylate

NB Narrow band

NBCK Narrow band correlated-k

\section{Introduction}

In the last decade k-distribution methods, such as the narrow band correlated-k (NBCK) model, the full-spectrum k-distribution (FSK) model and their extensions, have emerged as the most promising methods for engineering radiative heat transfer calculations due to their good accuracy and efficiency [1-4]. Their capability to model radiative heat transfer within 
the fuel rich core of pool fires was assessed by comparison with the exact line-by-line solutions, showing that they provide much better predictions than the methods currently used in fire simulators [5, 6]. In addition, in agreement with previous studies [7, 8], the radiative contribution of the gaseous fuel was found to play an important role in the radiative feedback toward the pool surface $[5,6]$.

A key prerequisite for the use of k-distribution methods in radiative heat transfer calculations in engineering applications is that a database of k-distributions must be available. Modest and co-workers $[9,10]$ demonstrated that the NB level is the best format to build such a database. This implies that, for each gas considered in the database, NB k-distributions must be stored for given sets of NBs, mole fractions, temperatures and pressures [10]. Storing kdistributions at the NB level provides several advantages, with the most important one being that accurate k-distributions at both NB and FS levels can be efficiently assembled for an arbitrary mixture of radiating gases and non-gray particles [9]. The NB k-distribution database can be built either directly from spectroscopic databases [10] or from NB transmissivity models, taking the advantage that the latter is the Laplace transform of the NB k-distribution function [11].

A NB database of k-distributions for carbon monoxide, carbon dioxide and water vapour can be generated [5-7, 9, 10] from HITEMP [12] and CDSD-1000 [13] spectroscopic databases, which were found to provide a good description of the line data over the range of temperatures encountered in combustion applications [14-16]. In order to apply this database to pool fire simulations it should be extended by adding k-distributions of fuels. HITRAN 2004 [17] was used to generate k-distributions for methane and ethylene [18, 19]. However, HITRAN was mainly developed for atmospheric applications and is expected to become inaccurate at temperatures higher than $600 \mathrm{~K}$ [10-22]. In addition, only a limited number of 
hydrocarbon fuels, such as methane, methanol, ethane, ethylene, and acetylene, is available in HITRAN. In the case of methane, Perrin and Soufiani [22] developed an extended spectroscopic database which was found able to provide band absorptances and total emissivities in good agreement with available experimental data up to $1500 \mathrm{~K}$. This database was used to derive NB parameters for the Lorentzian Malkmus statistical NB model [22 23]. Other well-known databases, such as the NIST [24] and the PNNL [24] databases, provide the absorption cross-sections of many species, but at low temperatures $\left(50^{\circ} \mathrm{C}\right)$ and are not appropriate for combustion and fire applications.

NB k-distribution functions can also be computed by considering the inverse Laplace transform of NB transmissivity [1, 11]. An analytical expression for the k-distribution function can be obtained when the Lorentzian Malkmus statistical NB model is considered [26]. The problem is then reduced to the determination of NB parameters. NB parameters were derived either from spectroscopic databases as done by the EM2C team [22, 23, 27] for carbon dioxide, water vapor, carbon monoxide, and methane or from NB transmissivity measurements (see $[28,29,16])$. In this paper, the second procedure was employed, since, as discussed previously, line-by-line data (HITRAN) are available only for a limited number of hydrocarbon fuels and can be inaccurate at temperatures relevant to fire and combustion applications. The medium resolution $\left(1 \mathrm{~cm}^{-1}\right)$ transmission measurements carried out at NIST up to $1000 \mathrm{~K}$ for nine fuels (methane, methanol, ethane, ethylene, propane, propylene, heptane, methyl methacrylate and toluene) $[7,30,31]$ are used to accomplish this task. These experimental results are used to extend the database of NB k-distributions developed in Refs [5, 6] for $\mathrm{CO}_{2}, \mathrm{H}_{2} \mathrm{O}$ and $\mathrm{CO}$ from CDSD-1000 [13] and HITEMP 2010 [32] spectroscopic databases to include these nine fuels by using the Wang and Modest procedure [10]. The paper is organized as follows: the experimental procedure and the experimental uncertainties are presented in section 2. Section 3 describes the theoretical framework used to obtain the 
NB parameters from measurements and to generate the k-distribution database. The results are presented and discussed in Section 4 with the accuracy of the NB database, the EM2C NB database [22, 23], and HITRAN 2012 [33] evaluated. Finally, Section 5 summarizes the conclusions drawn from this study.

\section{Experiments}

The medium resolution transmission measurements performed at NIST for nine fuels, namely methane, methanol, ethane, ethylene, propane, propylene, heptane, toluene and methyl methacrylate (MMA) [7, 30, 31], constitutes the starting point of this study. The experimental setup is briefly described in Section 2.1 whereas experimental uncertainties are estimated in Section 2.2. More details about the experimental setup and the experimental procedure can be found in Ref. [30].

\subsection{Experimental set up and procedures}

A Mattason Galaxy 7020 FTIR was used to measure transmissivities over a spectral interval ranging from $700 \mathrm{~cm}^{-1}$ to $4000 \mathrm{~cm}^{-1}$ at a resolution of $1 \mathrm{~cm}^{-1}$. The signal was averaged over 128 scans. The IR beam passed through a $31.75 \mathrm{~cm}$ cell and into an external Mercury Cadmium Telluride (MCT) detector (M10465 Graseby Infrared). The total pressure, $p$, was 1 atm. A mixture of fuel and nitrogen flowed through the cell, the flow rates of $\mathrm{N}_{2}$ being adjusted to provide the desired fuel mole fraction within $\pm 2 \% \Delta x / x= \pm 2 \%$. Typically, three or four pressure path lengths ranging from about $1.6 \times 10^{-3}$ to $1.6 \mathrm{~atm} \cdot \mathrm{cm}$ were considered. The cell was located inside a furnace. The set point was adjusted to maintain the inlet and outlet gas line temperature within $5 \mathrm{~K}(\Delta T=5 K)$. For each fuel, seven temperatures in the range from $295 \mathrm{~K}$ to $1000 \mathrm{~K}$ were considered, with $1000 \mathrm{~K}$ being close to the temperature $(\sim 1100 \mathrm{~K})$ at which the fast pyrolysis of most hydrocarbons occurs. 
The temperature of the windows was kept below $700 \mathrm{~K}$ to prevent oxidation of $\mathrm{ZnSe}$ by directing a jet of $\mathrm{N}_{2}$ on the outside of the windows. The uncertainty in variations of the absorption coefficient due to temperature variations near the cooled windows, $\Delta \kappa_{e} / \kappa_{e}$ was estimated to be $\pm 4 \%, \pm 5 \%$, and $\pm 6 \%$ for $600 \mathrm{~K}, 800 \mathrm{~K}$, and $1000 \mathrm{~K}$, respectively [30]. Table 1 summarizes the conditions of measurements for each fuel.

\subsection{Experimental uncertainties}

The experimental errors on the measured transmissivities are analyzed following the procedure described in Refs. [14-16]. The measured NB absorption coefficient $\bar{\kappa}_{e}$ is defined as:

$$
\bar{\tau}_{e}=\exp \left(-\bar{\kappa}_{e} x p l\right)
$$

where $\bar{\tau}_{e}$ is the experimental NB transmissivity, $x$ is the fuel mole fraction, $p$ is the total pressure and $l$ is the path length. The uncertainty in transmissivity results from uncertainties on mole fraction, $\Delta x / x$, uncertainties on temperature, $\Delta T / T$, and uncertainties on the absorption coefficient due to temperature variation near the cooled windows, $\Delta \kappa_{e} / \kappa_{e}$. The relative error on the path length is estimated to be negligible. The uncertainties on line intensity and line shape profile caused by temperature uncertainty were found to have an imperceptible influence on the NB absorption coefficient. As a consequence, the temperature uncertainty are assumed to affect NB absorption coefficient only through gas density and the root mean square relative error on transmissivity can be expressed as [14-16]:

$$
\sigma_{e}=\frac{\Delta \bar{\tau}_{e}}{\bar{\tau}_{e}}=-\log \bar{\tau}_{e} \sqrt{\left(\frac{\Delta \bar{\kappa}_{e}}{\bar{\kappa}_{e}}\right)^{2}+\left(\frac{\Delta T}{T}\right)^{2}+\left(\frac{\Delta x}{x}\right)^{2}}
$$




\section{Narrow-band k-distribution from Malkmus Model}

\subsection{Theory}

NB models are based on the discretization of the spectrum into relatively small spectral intervals (typically between 5 to $50 \mathrm{~cm}^{-1}$ ), $\Delta \eta$, over which the Planck function and the radiative properties of particles and walls are essentially constant. The Malkmus model is recognized as the most suitable statistical NB model for radiating gases. In this model, the line probability distribution is assumed to have the following expression [1]:

$$
P(S)=\frac{1}{S \ln R}\left[\exp \left(-S / S_{m}\right)-\exp \left(-R S / S_{m}\right)\right]
$$

where $S_{m}$ is the maximum value of the line intensity and $R$ is the ratio of the maximum to minimum value of the line intensity. $P(S)$ is the probability density function and thus satisfies $\int_{0}^{\infty} P(S) d S=1$. The NB averaged transmissivity is then expressed as [1]:

$$
\bar{\tau}_{\Delta \eta}=\exp \left[-\frac{\beta_{M}}{\pi}\left(\sqrt{1+\frac{2 \pi x p l \bar{\kappa}_{M}}{\beta_{M}}}\right)\right]
$$

where the parameters of the NB model, $\overline{\kappa_{M}}$ and $\beta_{M}$, represent the pressure based absorption coefficient and mean line width to the line spacing ratio, respectively. $\overline{\kappa_{M}}$ depends in the first order approximation only on temperature [27] and according to Ref. [16] $\beta_{M}$ is expected to depend only on the fuel mole fraction, $x$, as:

$\beta_{M}=x \beta_{M S}+(1-x) \beta_{M A}$

where $\beta_{M S}$ and $\beta_{M A}$ are the self and the air broadening parameters, respectively. For a given temperature, the NB averaged transmissivity can be computed from Eq. 4 provided that the three model parameters are known. 
The averaged NB transmissivity can also be computed from the NB k-distribution function or from the cumulative NB k-distribution function as [1]:

$$
\bar{\tau}_{\Delta \eta}=\int_{0}^{\infty} f(k) \exp (-k l) d k=\int_{0}^{1} \exp [-k(g) l] d g
$$

where $f(k)$ is the NB k-distribution function and $g(k)=\int_{0}^{k} f\left(k^{\prime}\right) d k^{\prime}$ is the NB cumulative kdistribution function. Eq. 6 shows that the NB transmissivity is the Laplace transform of the absorption coefficient probability density function $f(k) . f(k)$ can be then deduced from the transmissivity given by the Malkums model by considering an inverse Laplace transform of Eq. 4. The following analytical expression was obtained by Domoto [26]:

$$
f(k)=\frac{1}{2 \pi k}\left(\frac{2 \beta_{M} x p \bar{\kappa}_{M}}{k}\right)^{1 / 2} \exp \left[\frac{\beta_{M}}{2 \pi}\left(2-\frac{x p \bar{\kappa}_{M}}{k}-\frac{k}{x p \bar{\kappa}_{M}}\right)\right]
$$

Eq. 7 can be integrated to give the cumulative density function [11]:

$$
g(k)=\frac{1}{2}\left[1-\operatorname{erf}\left(\frac{a}{\sqrt{k}}-b \sqrt{k}\right)\right]+\frac{1}{2}\left[1-\operatorname{erf}\left(\frac{a}{\sqrt{k}}+b \sqrt{k}\right)\right]
$$

with $a=\sqrt{\frac{\beta_{M} x p \overline{\kappa_{M}}}{2 \pi}}$ and $b=\sqrt{\frac{\beta_{M}}{2 \pi x p \overline{\kappa_{M}}}}$. Although an analytical expression for $k(g)$ does not exist, it can be found numerically by using a Newton-Raphson iteration method. In general only few iterations are required when $k_{\max }$ (where $f(k)$ peaks) is used as the initial value of $k$ [11].

The NB parameters for each NB and for a set of temperatures allow generating a database of NB $k(g)$ distributions. $k(g)$ distributions can be stored efficiently by considering sets of mole fractions and temperatures and by using the fixed g-concept introduced by Wang and 
Modest [10]. Typically, mole fraction and temperature intervals of 0.25 and $100 \mathrm{~K}$ are used [10]. The fixed-g concept consists in considering a fixed set of g-values (typically 128) corresponding to abscissas of a Gaussian quadrature scheme [10]. This allows the determination of accurate mixed k-distributions for a gas mixture from the k-distributions of constituents contained in the database by using the mixing and assembly procedures given by Modest and Riazzi [9]. In addition, for arbitrary species mole fractions and temperatures, the NB absorption coefficient for each band can be computed from the database by carrying out linear interpolations on $x$ and spline interpolations on $T$ [10]. In the present study, the NB parameters for the Malkmus model for each fuel are determined from the measured transmissivity for a set of temperatures in the range $295 \mathrm{~K}-1000 \mathrm{~K}$ (see Table 1). The resolution proposed by Wang and Modest [10] and adopted in Ref. [5, 6] is considered ( see Table 2).

\subsection{Determination of the narrow band parameters}

The resolution of experimental transmissivities is reduced to that of the NB database. The transmissivity for the required band is then computed as a weighted sum of the experimental transmissivities as [16]:

$$
\bar{\tau}_{\Delta \eta}=\sum \frac{F \Delta \eta_{e}}{\Delta \eta} \bar{\tau}_{e}
$$

where $F$ is the fraction of the experimental NB which falls to the required NB of the NB database, $\Delta \eta_{e}$ is the resolution of the experimental data $\left(1 \mathrm{~cm}^{-1}\right)$ and $\Delta \eta$ is the resolution of the NB database, i.e., $25 \mathrm{~cm}^{-1}$ on the spectral interval of interest ranging from $700 \mathrm{~cm}^{-1}$ to $4000 \mathrm{~cm}^{-1}$ (see Table 2). The experimental standard deviations may also be weighted to estimate an error for $\bar{\tau}_{\Delta \eta}[16]$ : 


$$
\sigma_{\Delta \eta}=\frac{\Delta \bar{\tau}_{\Delta \eta}}{\bar{\tau}_{\Delta \eta}}=\sum \frac{F \Delta \eta_{e}}{\Delta \eta} \sigma_{e}
$$

$\bar{\tau}_{\Delta \eta}$ were used to obtain NB Malkmus parameters.

Two methods can be used to optimize the parameters. In the first, the parameter values are optimized by matching the curve of growth for each optical thickness. In the second, the absorption coefficient, $\overline{\kappa_{M}}$, is adjusted in the optically thin limit, whereas $\beta_{M S}$ and $\beta_{M A}$ are obtained by using a nonlinear least square fitting of the curve of growth for each optical thickness [27]. The second method is used in the present study.

Table 1 shows that the measurements were carried out for three or four rather weak fuel partial pressure path lengths. In order to improve the generality of the NB parameters, the number of reduced transmissivities to perform the fitting procedure was extended by extrapolating measured transmissivities to a wider range of pressure path lengths. Typically, one hundred pressure path lengths ranging from $10^{-3}$ to $100 \mathrm{~atm} \cdot \mathrm{cm}$ were considered to determine the NB parameters. The extrapolation procedure was based on the assumption that the pressure-based absorption coefficient, $\bar{\kappa}_{e}$, is weakly dependent on the fuel mole fraction. As a consequence, $\bar{\kappa}_{e}$ can be determined from the measured NB transmissivities and used to extrapolate the measurements to other pressure path lengths:

$$
\bar{\kappa}_{e}=-\log \bar{\tau}_{e} / x p l
$$

and

$$
\bar{\tau}_{e x t}=\exp \left(-\bar{\kappa}_{e} x_{e x t} p l_{e x t}\right)
$$

where $\bar{\tau}_{\text {ext }}$ represent the extrapolated transmissivity with a resolution of $1 \mathrm{~cm}^{-1}$ corresponding to the extrapolated fuel partial pressure path length. The accuracy of this procedure is 
examined in Fig. 1, which represents $\bar{\tau}_{\Delta \eta}$ for methane at temperatures of $297 \mathrm{~K}$ and $1000 \mathrm{~K}$. For each $\mathrm{NB}, \bar{\kappa}_{e}$ was determined from a transmissivity with a resolution of $1 \mathrm{~cm}^{-1}$ computed from HITRAN 2012 [33] for $x=0.0028$. Figure 1 shows that the extrapolated transmissivities determined from $\bar{\kappa}_{e}$ are in overall good agreement with those determined directly from HITRAN 2012 [33], demonstrating the validity of the extrapolation procedure. Nevertheless, larger discrepancies are observed for $x=0.9$ than for $x=0.05$, which shows that the extrapolation procedure becomes increasingly inaccurate for large pressure path length extension beyond the measurement conditions.

\section{Results and discussions}

\subsection{Comparison of calculated transmissivities with reduced experimental data}

Figures 2-10 show a comparison between the NB transmissivities calculated from the Malkmus NB parameters developed in this study and the reduced experimental data for methane, methanol, ethane, ethylene, propane, propylene, heptane, MMA and toluene, respectively. Methane, methanol, ethane and ethylene are included in the HITRAN spectroscopic database [33]. For these molecules, the reduced NB transmissivities were also computed from the line data of HITRAN 2012 [33] at the same resolution as the NB database $\left(25 \mathrm{~cm}^{-1}\right)$. In these calculations, the line shape profile was assumed to follow a Lorentz profile when the ratio between the collision broadening line half-width and the Doppler line half-width is greater than 5. When this condition is not satisfied a Voigt profile is considered [34].

These transmissivities are plotted in Figs. 2-5. For methane, NB transmissivities calculated with the EM2C database [22, 23] are also included for comparison (see Fig. 2). Although the spectral resolution for these transmissivities is also $25 \mathrm{~cm}^{-1}$, it is important to notice that the centers of the NBs are shifted by $12.5 \mathrm{~cm}^{-1}$ as compared to those in the present NB database. 
Figures 2 to 10 show that the calculated transmissivities reproduce very well the experimental data on the whole, demonstrating the quality of the fit. For methane, transmissivities computed with HITRAN 2012 are in reasonable agreement with the experimental data for temperatures up to $600 \mathrm{~K}$ (see Fig. 2). Figure 2c shows that HITRAN 2012 significantly overestimates the transmissivities compared to the experimental data at1000 K, which is consistent with the conclusions reported in [22]. HITRAN 2012 does not include all the hot lines necessary to predict accurately high temperature spectra, explaining the observed discrepancies. Figure $2 \mathrm{c}$ shows also clearly the improvements provided by the EM2C database at $1000 \mathrm{~K}$.

In order to evaluate the $\mathrm{EM} 2 \mathrm{C}$ database in a proper manner, the transmissivities displayed in Fig. 11 are computed by using its spectral discretization [22, 23]. At $297 \mathrm{~K}$, the excellent accordance between the transmissivities determined from HITRAN 2012 and the experimental data suggests the good accuracy of HITRAN 2012 at this temperature. The EM2C database also provides predictions in very good agreement with the experimental data, despite somewhat overpredictions of $2.7 \%$ and $6.8 \%$ at $1300 \mathrm{~cm}^{-1}$ and $3025 \mathrm{~cm}^{-1}$, respectively. At $600 \mathrm{~K}$, the agreement between the experimental data and the two databases is found to be excellent for all the NBs. Nevertheless, some small discrepancies of the order of $2 \%$ between, on the one hand, both experimental data and HITRAN 2012 and, on the other hand, the EM2C database are still observed. As expected from the previous studies [22, 23], the EM2C database predictions display a much better agreement with the experiments at 1000 K than those obtained with HITRAN 2012. A very good agreement between the experiments and the EM2C is obtained for the $v_{3}$ band $\left(\mathbf{2 7 0 0 - 3 3 0 0 ~} \mathbf{c m}^{-\mathbf{1}}\right)$, despite the localized discrepancies of the order of $26 \%$ at $3000 \mathrm{~cm}^{-1}$. In the $v_{4}$ band, the transmissivities computed with the EM2C database are also found to overestimate the experimental data. At these temperatures, measurements of transmissivities are challenging and it is reasonable to believe 
that their accuracy is reduced. In particular, the cooling of the windows to prevent oxidation induces a non-uniform profile of temperatures in the cell, with temperatures near the windows significantly lower than those imposed in the cell [30]. In the $v_{4}$ band transmissivities were found to be sensitive to temperature and to increase as the temperature decreases. This can, then, explain that the EM2C calculations underestimate the data since the non-uniformity of the temperature is not taken into account in the simulations.

For methanol the line data of HITRAN 2012 are only available for the C-O stretching band (around $1033 \mathrm{~cm}^{-1}$ ). Figure 3 shows that the predicted transmissivities for this band are in reasonable agreement with the experimental data at $293 \mathrm{~K}$, but become less and less accurate as the temperature increases. Figure 4 displays the computed and experimental transmissivities for ethane. HITRAN 2012 predicts accurately the transmissivity in the range from 600 to $1800 \mathrm{~cm}^{-1}$ at a temperature of $296 \mathrm{~K}$, but large discrepancies are observed at 600 and $1000 \mathrm{~K}$. In addition, Fig. $4 \mathrm{a}_{2}$ shows that HITRAN 2012 provides poor predictions of the transmissivity for the $\mathrm{CH}_{3}$ stretching band even at room temperature (around $3000 \mathrm{~cm}^{-1}$ ). Comparisons between the transmissivities computed with HITRAN 2012 and the experimental data for ethylene depict a similar overall behavior as that for the other fuels (see Fig. 5). A reasonable agreement is observed at room temperature (see Figs. 5a $a_{1}-a_{3}$ ). However, large discrepancies are encountered at higher temperatures.

\subsection{Accuracy of the NB database}

Figures 2 to 10 show that the calculated transmissivities reproduce accurately the experimental data from which the present Malkmus NB parameters are derived. However, as shown in Fig.1, the extrapolation procedure can lead to larger discrepancies for values of the fuel partial pressure path length outside the range of measurements. 
The objective of this section is to assess the accuracy of the NB database. Methane is considered to accomplish this task due to the availability of, on the one hand, a significant amount of experimental data and, on the other hand, the EM2C NB database that can be used as a reference in view of the results reported in Ref. [22]. This assessment is performed by comparing the integrated radiative properties computed with the present NB database to those computed with the EM2C database and experimental data. These integrated properties were also computed from HITRAN 2012 and from the pressure-based absorption coefficient, $\bar{\kappa}_{e}$, deduced from the NIST experimental data (see Eq. 11). The data obtained with the latter model will be referred to as NIST database hereafter. It is important to keep in mind that this denomination concerns the medium resolution transmission measurements performed by Wakatsuki and co-workers [7, 30, 31] and not the database of absorption cross-section [24].

Figure 12 shows the total emissivities, defined as $\varepsilon=\frac{1}{I_{b}(T)} \int_{0}^{\infty}\left(1-\exp \left(-\kappa_{\eta} l\right)\right) I_{b \eta}(T) d \eta$, as a function of the temperature for different pressure path lengths. The computed emissivities are compared with the data of Brosmer and Tien [35]. As expected, the total emissivities computed with the EM2C database are in good accordance with the experimental data whereas those computed with HITRAN 2012 underestimate significantly the total emissivity above $600 \mathrm{~K}$. It is also expected that the current NB database provides emissivities in close agreement with those obtained using the absorption coefficients derived from the NIST measurements. This demonstrates that the NB database has the same accuracy as the NIST database from which the Malkmus NB parameters were derived. Comparison with the EM2C database shows that the NB database and the NIST emissivities exhibit the largest discrepancies for a fuel partial pressure path length of $2 \mathrm{~cm} \cdot$ atm and temperatures lower than $600 \mathrm{~K}$. Relative errors of $22 \%$ and $30 \%$ are observed at $297 \mathrm{~K}$ and $500 \mathrm{~K}$, respectively. Regardless of the path lengths considered, both 
the NB and the NIST databases are found to be more accurate than HITRAN 2012 for temperatures greater than $600 \mathrm{~K}$.

Figure 13 shows the band absorbance for the $v_{4}\left(800-2000 \mathrm{~cm}^{-1}\right)$ and the $v_{3}\left(2000-3500 \mathrm{~cm}^{-1}\right)$ bands defined as $\alpha=\int_{b a n d, i}\left(1-\exp \left(-\kappa_{\eta} l\right)\right) d \eta$. The experimental data correspond to the measurements of Lee and Happel [36]. The observations for the band absorbance shown in Fig. 13 are overall similar to those for the total emissivities shown in Fig. 12. The EM2C database provides accurate absorbtances for all the temperatures considered, whereas HITRAN 2012 becomes significantly inaccurate above $600 \mathrm{~K}$. The absorbtances predicted from the present NB and NIST databases are in close agreement and present a substantial improvement over the HITRAN 2012 results above $600 \mathrm{~K}$. Comparisons with the EM2C absorbtances show that the largest relative errors are about $14 \%$ for the $v_{3}$ region and $30 \%$ for the $v_{4}$ region and occur at temperature of $297 \mathrm{~K}$ and $400 \mathrm{~K}$, respectively.

Figure 14 shows the pressure-based Planck-mean absorption coefficient of methane. Results from the EM2C database and HITRAN 2012 are in good agreement up to $600 \mathrm{~K}$. Above this temperature HITRAN 2012 starts to underestimate significantly the Planck-mean absorption coefficient due to the absence of hot lines. The Tien's data were obtained by integrating the exponential wide band data across the spectrum, based on correlations of lowresolution experiments [37]. The data of Tien are higher than the EM2C results by about 30\%. Results of the present NB and NIST databases are in relatively good agreement. Both databases provide predictions in better agreement with the EM2C data than the Tien correlation and are more accurate than HITRAN 2012 above $600 \mathrm{~K}$. Results of the present NB and NIST databases are within $20 \%$ of the EM2C Planck-mean absorption coefficients. 
Radiative heat transfer calculations are conducted along two non-isothermal nonhomogeneous paths involving methane concentration and temperature representative of those encountered along the axis of a pool fire (see Figs. $15 a_{1}$ and $b_{1}$ ). The temperatures and methane concentrations were taken from the simulations reported in $[5,38]$, where two methane turbulent buoyant diffusion flames, generated on a burner $0.38 \mathrm{~m}$ in diameter with heat release rates of 33.4 and $175.8 \mathrm{~kW}$ were considered. The optical thicknesses, based on the Planck mean absorption coefficient, are 0.25 and 1.87 for the 34 and $176 \mathrm{~kW}$ pool fires, respectively. Figs. $15 a_{2}$ and $b_{2}$ show the axial evolution of the integrated radiative intensity. In both cases, very large discrepancies were observed between EMC2 and HITRAN 2012 predictions, confirming that HITRAN 2012 is not suitable for such kind of applications. The predicted radiative intensities calculated with NIST and the present NB databases are very similar and the comparisons with the EM2C calculations show that they are significantly more accurate than the HITRAN 2012 predictions. For the $34 \mathrm{~kW}$ pool fire, a very good agreement is observed with the EM2C computations (see Fig. $15 \mathrm{a}_{2}$ ). For the $176 \mathrm{~kW}$ pool fire, the absorption of methane between $\mathrm{z}=0.5 \mathrm{~m}$ and the burner exit is overpredicted, which result in a maximum relative error of the order of $35 \%$ at the burner exit.

It should be pointed out that the spectral resolution of the NIST database is too low to be used directly in CFD calculations. In fire simulators, these data are generally used to generate the Planck mean-absorption coefficients which are used as radiative properties for the radiatively participating gases in the radiative transfer equation $[39,40]$. Calculations with the Planck mean absorption coefficients computed from the NIST database are referred to as 'NIST Planck' in Fig. $15 \mathrm{a}_{2}$ and $\mathrm{b}_{2}$. It can be observed that the discrepancies obtained by using the Planck mean absorption coefficients are significantly greater than those observed for the present NB and NIST databases. As expected, these discrepancies are found to increase as the optical thickness increases. 


\section{Conclusions}

A database of Malkmus NB parameters for nine fuels, namely methane, methanol, ethane, ethylene, propane, propylene, heptane, MMA, and toluene was generated from the NIST transmissivity measurements for temperatures ranging between $295 \mathrm{~K}$ and $1000 \mathrm{~K}$. The accuracy of this database was evaluated by comparisons with experimental data and with the EM2C NB database for methane and the spectroscopic database HITRAN 2012 for methane, methanol, ethane and ethylene. The following conclusions can be drawn:

1) The EM2C NB database is recommended for radiative transfer calculations involving methane.

2) HITRAN 2012 gives rise to large error at temperatures above $600 \mathrm{~K}$. In addition, this database does not include some important bands for methanol and ethylene.

3) The Malkmus NB database developed in this study has the same accuracy as the NIST database from which the Malkmus NB parameters were derived. These databases predict spectrally-integrated radiative properties within about $20 \%$ of measurements, showing that they can be used for engineering applications.

4) Applications to radiation calculations along lines of sight with methane concentrations and temperatures representative of pool fires suggest that both the Malkmus narrow band and NIST databases are accurate for small scale pool fires. For larger scale pool fires, however, relative errors of about $30 \%$ are observed.

5) The use of Planck-mean absorption coefficient should be avoided if accurate predictions are required.

As a final remark, it is important to point out that the accuracy of the Malkmus NB database can be substantially improved if the measured transmissivity were available for a wider range of fuel partial pressure-pathlength product than the current NIST database. The NB 
parameters derived in this study can be readily used to generate a NB k-distribution database based on the Malkmus NB model.

The database of Malkmus NB parameters described in this study is available upon request.

\section{Acknowledgments}

The authors wish to thank Drs. V. Lecoustre, G. Jackson, and A. Hamins of NIST for providing the medium resolution transmission data and for their assistance in using them.

\section{References}

[1] M. F. Modest, Radiative Heat Transfer, Academic Press, 2003.

[2] V. Goutiere, F. Liu, A. Charrette, An assessment of real-gas modeling in 2D enclosures, J. Quant. Spectrosc. Radiat. Transfer 64 (2000) 299-326.

[3] P. J. Coelho, Numerical simulation of radiative heat transfer from non-gray gases in threedimensional enclosures, J. Quant. Spectrosc. Radiat. Transfer 74 (2002) 307-328.

[4] R. Demarco, J. L. Consalvi, A. Fuentes, S. Melis, Assessment of radiative property models in non-gray sooting media, Int. J. Thermal Sci. 50 (2011) 1672-1684.

[5] J.L. Consalvi, F. Liu, Radiative Heat transfer Through the Fuel Rich Core of LaboratoryScale pool Fire, Combust. Sci. Technol.186 (2014) 475-489.

[6] J.L. Consalvi, F. Liu, Radiative Heat Transfer within the Fuel Rich Core of Pool FiresPart I: Axisymmetric Calculations with the Line-By-Line Method and Approximate Property Models in Laboratory-Scale Methane Fires, Int. J. Thermal Sci. 84 (2014) 104117. 
[7] K. Wakatsuki, G. S. Jackson, A. Hamins, M. R. Nyden, Effects of fuel absorption on radiative heat transfer in methanol pool fires, Proc. Combust. Inst. 31 (2007) 2573-2580.

[8] F. Jiang, J. L. de Ris, H. Qi, M. M. Khan, Radiation blockage in small scale PMMA combustion, Proc. Comb. Inst. 33 (2011) 2657-2664.

[9] M. F. Modest, R. J. Riazzi, Assembly full spectrum $k$-distribution from a narrow band database: effects of mixing gases, gases and non-gray absorbing particles and non-gray scatters in non-gray enclosures, J. Quant. Spectrosc. Radiat. Transfer 90 (2005) 169-189.

[10] L. Wang, M. F. Modest, Narrow-band based multiscale full-spectrum k-distribution method for radiative transfer in inhomogeneous gas mixtures, J. Heat Transfer 127 (2005) 740-748.

[11] A. A. Lacis, V. A. Oinas, Description of the correlated-k distribution method for modeling nongray gaseous absorption, thermal emission, and multiple scattering in vertically inhomogeneous atmospheres, J. Geophys. Res. 96 (1991) 9027-9063.

[12] L. S. Rothman, C. Camy-Peyret, J. M. Flaud, R. Gamache, A. Goldman, D. Goorvitch, R. Hawkins, J. Schroeder, J. Selby, R. Wattson, HITEMP, the high-temperature molecular spectroscopic database, J. Quant. Spectrosc. Radiat. Transfer 60 (1998):665-710.

[13] S. A. Tashkun, V. I. Perevalov, J. L. Teffo, A. D. Bykov, N. N. Lavrentieva, CDSD1000, the high-temperature carbon dioxide spectroscopic databank, J. Quant. Spectrosc. Radiat. Transfer 82 (2003) 165-196.

[14] S. P. Bharadwaj, M. F. Modest, R. Riazzi, Medium resolution transmission measurements of water vapor at high temperature, J. Heat Transfer 128 (2006) 374-381.

[15] S. P. Bharadwaj, M. F. Modest, Medium resolution transmission measurements of $\mathrm{CO}_{2}$ at high temperature - an update', J. Quant. Spectrosc. Radiat. Transfer 103 (2007). 146155. 
[16] S. P. Bharadwaj, Medium resolution transmission measurements of $\mathrm{CO}_{2}$ and $\mathrm{H}_{2} \mathrm{O}$ at high temperature and a multiscale Malkmus model for treatment of inhomogeneous gas paths, Phd. Thesis, The Pennsylvania State University, 2005.

[17] L.S. Rothman, D. Jacquemart, A. Barbe, D. Chris Benner, M. Birk, L. R. Brown, M. R. Carleer, C. Chackerian Jr., K. Chance, L. H. Coudert, V. Dana, V. M. Devi, J. M. Flaud, R. R. Gamache, A. Goldman, J. M. Hartmann, K. W. Jucks, A. G. Maki, J. Y. Mandin, S. T. Massie, J. Orphal, A. Perrin, C. P. Rinsland, M. A. H. Smith, J. Tennyson, R. N. Tolchenov, R.A. Toth, J. Vander Auwera, P. Varanasi, G. Wagner, The HITRAN 2004 molecular spectroscopic database, J. Quant. Spectrosc. Radiat. Transfer 96 (2005) 139-204.

[18] G. Pal, M. F. Modest, A narrow band-based multiscale multigroup full-spectrum kdistribution method for radiative transfer in nonhomogeneous gas-soot mixtures, J. Heat Transfer 132 (2010) 1-10.

[19] G. Pal, M. F. Modest, A multiscale full spectrum k-distribution method for radiative heat transfer in nonhomogeneous gas-soot mixtures with wall emission, Comput. Thermal Sci. 1 (2009) 137-158.

[20] M. F. Modest, S. P. Bharadwaj, Medium-resolution, high-Temperature transmissivity measurements and correlations for carbon dioxide-nitrogen mixtures", J. Quant. Spectrosc. Radiat. Transfer 73 (2002) 329-338.

[21] H. Chu, F. Liu, H. Zhou, Calculations of gas thermal radiation transfer in onedimensional planar enclosure using LBL and SNB models, Int. J. Heat Mass Transfer 54 (2011) 4736-4745.

[22] M. Y. Perrin, A. Soufiani, Approximate radiative properties of methane at high temperature, J. Quant. Spectrosc. Radiat. Transfer 103 (2007) 3-13. 
[23] P. Rivière, A. Soufiani, Updated band model parameters for $\mathrm{H}_{2} \mathrm{O}, \mathrm{CO}_{2}, \mathrm{CH}_{4}$ and $\mathrm{CO}$ radiation at high temperature, Int. J. Heat Mass Transfer 55 (2012) 3349-3358.

[24] P. M. Chu, F. R. Guenter, G. C. Rhoderick, W. J. Lafferty, The NIST quantitative infrared database, J. Res. Natl. Inst. Stand. Technol. 104 (1999) 59-81.

[25] S. W. Sharpe, T. J. Johnson, R. L. Sams, P. M. Chu, G. C. Rhoderick, P. A. Johnson, Gas-phase databases for quantitative infrared spectroscopy. Appl. Spectrosc. 58 (2004) $1452-1461$.

[26] G. A. Domoto, Frequency integration for radiative transfer problems involving homogeneous non-gray gases: the inverse transmission function, J. Quant. Spectrosc. Radiat. Transfer 14 (1974) 935-942.

[27] A. Soufiani, J. Taine, High temperature gas radiative property parameters of statistical narrow-band model for $\mathrm{H}_{2} \mathrm{O}, \mathrm{CO}_{2}$ and $\mathrm{CO}$ and correlated-k model for $\mathrm{H}_{2} \mathrm{O}$ and $\mathrm{CO}_{2}$, Int. $\mathrm{J}$. Heat Mass Transfer 40 (1997) 987-991.

[28] W. J. Phillips, Band model parameters for the $2.7 \mu \mathrm{m}$ band of $\mathrm{H}_{2} \mathrm{O}$, J. Quant. Spectrosc. Radiat. Transfer 43 (1990) 13-31.

[29] W. J. Phillips, Band-model parameters for $4.3 \mu \mathrm{m} \mathrm{CO} 2$ band in the $300-1000 \mathrm{~K}$ temperature region, J. Quant. Spectrosc. Radiat. Transfer 48 (1992) 91-104.

[30] K. Wakatsuki, High temperature radiation absorption of fuel molecules and an evaluation of its influence on pool fire modeling, Phd. Thesis, University of Maryland, 2005.

[31] K. Wakatsuki, S. P. Fuss, A. Hamins, M. R. Nyden, A technique for extrapolating absorption coefficient measurements to high temperatures, Proc. Combust. Inst. 30 (2005) $1565-1573$.

[32] L. S. Rothman, I. E. Gordon, R. J. Barber, H. Dothe, R. R. Gamache, A. Goldman, V. I. Perevalov, S. A. Tashkun, J. Tennyson, HITEMP: the high-temperature molecular, J. Quant. Spectrosc. Radiat. Transfer 111 (2010) 2139-2150. 
[33] L. S. Rothman, I. E. Gordon, Y. Babikov, A. Barbe, D. Chris Benner, P. F. Bernath, M. Birk, L. Bizzocchi, V. Boudon, L. R. Brown, A. Campargue, K. Chance, E. A. Cohen, L. H. Coudert, V. M. Devi, B. J. Drouin, A. Fayt, J. M. Flaud, R. R. Gamache, J. J. Harrison, J. M. Hartmann, C. Hill, J. T. Hodges, D. Jacquemart, A. Jolly, J. Lamouroux, R. J. Le Roy, G. Li, D. A. Long, O. M. Lyulin, C. J. Mackie, S. T. Massie, S. Mikhailenko, H. S. P. Müller, O. V. Naumenko, A. V. Nikitin, J. Orphal, V. Perevalov, A. Perrin, E. R. Polovtseva, C. Richard, The HITRAN2012 molecular spectroscopic database, J. Quant. Spectrosc. Radiat. Transfer 130 (2013) 4-50.

[34] J. Taine, A. Soufiani, Gas IR radiative properties: from spectroscopic data to approximate models, Adv. Heat Transfer 33 (1999) 295-415.

[35] M. A. Brosmer, C. L. Tien, Infrared radiation properties of methane at elevated temperatures J. Quant. Spectrosc. Radiat. Transfer 33 (1985) 521-532.

[36] R. H. C. Lee, J. Happel, Thermal radiation of methane gas, I\&EC Fundam 3 (1964) $167-76$.

[37] C.L. Tien, Thermal radiation properties of gases, Adv. Heat Transfer 5 (1968) 253324.

[38] J. L. Consalvi, R. Demarco, A. Fuentes, Modeling thermal radiation in buoyant turbulent diffusion flames, Combust. Theor. Model. 16 (2012) 817-841.

[39] V. Novozhilov, H. Koseki, CFD prediction of pool fire burning rates and flame feedback, Comb. Sci. Technol. 176 (2004) 1283-1307.

[40] S. Hostikka, K. B. McGrattan, A. Hamins, Numerical Modeling of Pool Fires Using LES an Finite Volume Method for Radiation, in: D.D. Evans (Ed.), Fire Safety ScienceProc. $7^{\text {th }}$ International Symposium on Fire Safety Science, Worcester, USA, 2003, pp. 383-394. 
Table 1. Experimental conditions [30]. For each fuel measurements were conducted for seven temperatures referred to as $T_{i}$ with $i=1$ to 7 . For each temperature, the first line indicates the value of the temperature whereas the following lines indicate the fuel concentrations in ppm.

\begin{tabular}{|c|c|c|c|c|c|c|c|}
\hline Fuel/Temperature & $T_{1}$ & $\overline{T_{2}}$ & $T_{3}$ & $T_{4}$ & $T_{5}$ & $\overline{T_{6}}$ & $\overline{T_{7}}$ \\
\hline \multirow{5}{*}{ Methane } & $297 \mathrm{~K}$ & $400 \mathrm{~K}$ & $450 \mathrm{~K}$ & $499 \mathrm{~K}$ & $600 \mathrm{~K}$ & $800 \mathrm{~K}$ & $1000 \mathrm{~K}$ \\
\hline & 491 & 491 & 491 & 491 & 491 & 24140 & 24140 \\
\hline & 1032 & 1032 & 1032 & 1032 & 1032 & 31838 & 31838 \\
\hline & 1933 & 1933 & 1933 & 1933 & 1933 & 39415 & 39415 \\
\hline & 2832 & 2832 & 2832 & 2832 & 2832 & 51937 & 51937 \\
\hline \multirow{4}{*}{ Methanol } & $293 \mathrm{~K}$ & $396 \mathrm{~K}$ & $443 \mathrm{~K}$ & $483 \mathrm{~K}$ & $570 \mathrm{~K}$ & $804 \mathrm{~K}$ & $1000 \mathrm{~K}$ \\
\hline & 1570 & 1590 & 1445 & 1540 & 1574 & 1692 & 1543 \\
\hline & 2256 & 2323 & 2235 & 2213 & 2286 & 2472 & 2302 \\
\hline & 2912 & 2935 & 2960 & 2857 & 2904 & 3193 & 3055 \\
\hline \multirow{4}{*}{ Ethane } & $296 K$ & $400 \mathrm{~K}$ & $400 \mathrm{~K}$ & $500 \mathrm{~K}$ & $600 \mathrm{~K}$ & $800 \mathrm{~K}$ & $1000 \mathrm{~K}$ \\
\hline & 2559 & 2559 & 2559 & 2559 & 2559 & 2559 & 2559 \\
\hline & 6632 & 6632 & 6632 & 6632 & 6632 & 6632 & 6632 \\
\hline & 10672 & 10672 & 10672 & 10672 & 10672 & 10672 & 10672 \\
\hline \multirow{4}{*}{ Ethylene } & $296 K$ & $400 \mathrm{~K}$ & $450 \mathrm{~K}$ & $500 \mathrm{~K}$ & $601 \mathrm{~K}$ & $801 \mathrm{~K}$ & $1000 \mathrm{~K}$ \\
\hline & 2470 & 2470 & 2470 & 2470 & 2470 & 2470 & 5660 \\
\hline & 5660 & 5660 & 5660 & 5660 & 5660 & 5660 & 9619 \\
\hline & 9619 & 9619 & 9619 & 9619 & 9619 & 9619 & 14328 \\
\hline \multirow{4}{*}{ Propane } & $295 \mathrm{~K}$ & $396 \mathrm{~K}$ & $435 \mathrm{~K}$ & $513 \mathrm{~K}$ & $578 \mathrm{~K}$ & $790 \mathrm{~K}$ & $1009 \mathrm{~K}$ \\
\hline & 1000 & 1000 & 1000 & 1000 & 1000 & 1000 & 1000 \\
\hline & 2500 & 2500 & 2500 & 2500 & 2500 & 2500 & 2500 \\
\hline & 4000 & 4000 & 4000 & 4000 & 4000 & 4000 & 4000 \\
\hline \multirow{4}{*}{ Propylene } & $296 K$ & $390 \mathrm{~K}$ & $444 K$ & $491 \mathrm{~K}$ & $594 \mathrm{~K}$ & $793 \mathrm{~K}$ & $1003 \mathrm{~K}$ \\
\hline & 5000 & 5000 & 5000 & 5000 & 5000 & 5000 & 5000 \\
\hline & 10000 & 10000 & 10000 & 10000 & 10000 & 10000 & 10000 \\
\hline & 15000 & 15000 & 15000 & 15000 & 15000 & 15000 & 15000 \\
\hline \multirow{4}{*}{ Heptane } & $293 \mathrm{~K}$ & $400 \mathrm{~K}$ & $450 \mathrm{~K}$ & $490 \mathrm{~K}$ & $593 \mathrm{~K}$ & $794 K$ & $1000 \mathrm{~K}$ \\
\hline & 474 & 458 & 480 & 474 & 479 & 468 & 548 \\
\hline & 985 & 951 & 982 & 964 & 992 & 952 & 971 \\
\hline & 1554 & 1500 & 1564 & 1561 & 1588 & 1389 & 1149 \\
\hline \multirow{4}{*}{ MMA } & $297 \mathrm{~K}$ & $396 K$ & $441 \mathrm{~K}$ & $483 \mathrm{~K}$ & $597 K$ & $803 K$ & $1014 K$ \\
\hline & 1716 & 1670 & 1600 & 1613 & 1716 & 1716 & 1716 \\
\hline & 2474 & 2413 & 2330 & 2474 & 2474 & 2474 & 2474 \\
\hline & 3162 & 3217 & 2978 & 3162 & 3162 & 3162 & 3162 \\
\hline \multirow{4}{*}{ Toluene } & $300 \mathrm{~K}$ & $396 \mathrm{~K}$ & $440 \mathrm{~K}$ & $477 \mathrm{~K}$ & $587 \mathrm{~K}$ & $795 \mathrm{~K}$ & $999 \mathrm{~K}$ \\
\hline & 2548 & 2548 & 2340 & 2617 & 2557 & 2627 & 2821 \\
\hline & 3183 & 3021 & 2666 & 3032 & 3026 & 3043 & 3260 \\
\hline & 4278 & 3929 & 3446 & 3964 & 3729 & 4021 & 4345 \\
\hline
\end{tabular}


Table 2. Nominal NB ranges for the NB database [10].

\begin{tabular}{|c|c|c|}
\hline Spectral Intervals $\left(\mathrm{cm}^{-1}\right)$ & Resolution $\left(\mathrm{cm}^{-1}\right)$ & Number of NBs \\
\hline $200-300$ & 10 & 10 \\
\hline $300-4000$ & 25 & 148 \\
\hline $4000-5000$ & 50 & 20 \\
\hline $5000-10000$ & 100 & 50 \\
\hline $10000-15000$ & 250 & 20 \\
\hline Total & & 248 \\
\hline
\end{tabular}




\section{List of Figure Captions}

Figure 1. Validity of the extrapolation procedure for methane for two temperatures of a) $297 \mathrm{~K}$ and b) $1000 \mathrm{~K}$. The indexes denote 1) the $v_{4}$ band and 2) the $v_{3}$ band.

Figure 2. Comparison between calculated transmissivities and reduced experimental transmissivities for methane for: a) $297 \mathrm{~K}$, b) $600 \mathrm{~K}$, and c) $1000 \mathrm{~K}$. The indexes denote 1) the $v_{4}$ band and 2) the $v_{3}$ band.

Figure 3. Comparison between calculated transmissivities and reduced experimental transmissivities for methanol for: a) $293 \mathrm{~K}$, b) $570 \mathrm{~K}$, and c) $1000 \mathrm{~K}$. The indexes denote 1) the C-O stretching and the $\mathrm{CH}_{3}$ bending bands and 2) the $\mathrm{CH}_{3}$ stretching and $\mathrm{O}-\mathrm{H}$ stretching bands.

Figure 4. Comparison between calculated transmissivities and reduced experimental transmissivities for ethane for: a) $296 \mathrm{~K}$, b) $600 \mathrm{~K}$, and c) $1000 \mathrm{~K}$. The indexes denote 1) the $\mathrm{CH}_{3}$ rock, the $\mathrm{C}-\mathrm{C}$ stretching and $\mathrm{CH}_{3}$ deform bands and 2) the $\mathrm{CH}_{3}$ stretching band.

Figure 5. Comparison between calculated transmissivities and reduced experimental transmissivities for ethylene for: a) $296 \mathrm{~K}$, b) $600 \mathrm{~K}$, and c) $1000 \mathrm{~K}$. The indexes denote 1) the $=\mathrm{CH}_{2}$ out of the plane bending band, 2) the $\mathrm{C}-\mathrm{H}$ in plane and out the plane bending bands and 3) the $\mathrm{CH}_{3}$ and $=\mathrm{CH}_{2}$ stretching bands.

Figure 6. Comparison between calculated transmissivities and reduced experimental transmissivities for propane for: a) $295 \mathrm{~K}$, b) $578 \mathrm{~K}$, and c) $1009 \mathrm{~K}$. The indexes denote 1) the $\mathrm{C}-\mathrm{H}$ bending band and 2) the C-H stretching band.

Figure 7. Comparison between calculated transmissivities and reduced experimental transmissivities for propylene for: a) $296 \mathrm{~K}$, b) $594 \mathrm{~K}$, and c) $1003 \mathrm{~K}$. The indexes denote 1) the $=\mathrm{CH}_{2}$ out of the plane bending band, 2) the $\mathrm{C}-\mathrm{H}$ in plane and out the plane bending bands and the $\mathrm{C}=\mathrm{C}$ stretching band and 3) the $\mathrm{CH}_{3}$ and the $=\mathrm{CH}_{2}$ stretching bands. 
Figure 8. Comparison between calculated transmissivities and reduced experimental transmissivities for heptane for: a) $295 \mathrm{~K}$, b) $593 \mathrm{~K}$, and c) $1000 \mathrm{~K}$. The indexes denote 1) the C-H bending band and 2) the C-H stretching band.

Figure 9. Comparison between calculated transmissivities and reduced experimental transmissivities for MMA for: a) $297 \mathrm{~K}$, b) $597 \mathrm{~K}$, and c) $1014 \mathrm{~K}$. The indexes denote 1) the $=\mathrm{CH}_{2}$ out of the plane bending band, 2) the $\mathrm{C}-\mathrm{O}$ stretching, the $\mathrm{CH}_{3}$ bending, the $\mathrm{C}=\mathrm{C}$ and $\mathrm{C}=\mathrm{O}$ stretching bands, and 3) the $\mathrm{CH}_{3}$ and the $=\mathrm{CH}_{2}$ stretching bands.

Figure 10. Comparison between calculated transmissivities and reduced experimental transmissivities for toluene for: a) $300 \mathrm{~K}$, b) $587 \mathrm{~K}$, and c) $999 \mathrm{~K}$. The indexes denote 1) the phenyl $=\mathrm{CH}$ out of the plane bending and the phenyl= $\mathrm{CH}$ in the plane bands, 2) the phenyl $\mathrm{C}=\mathrm{C}$ stretching band and 3) the $\mathrm{CH}_{3}$ and $=\mathrm{CH}$ stretching band.

Figure 11. Comparison between calculated transmissivities and reduced experimental transmissivities for methane for: a) $297 \mathrm{~K}$, b) $600 \mathrm{~K}$, and c) $1000 \mathrm{~K}$. The indexes denote 1) the $v_{4}$ band and 2) the $v_{3}$ band. The spectral discretization is that of the EM2C database.

Figure 12. Comparisons between total emissivities of methane calculated from the NB database, the NIST database, the EM2C database, HITRAN 2012 and the data from Brosmer and Tien [35] deduced from absorption measurements. Methane is assumed to be diluted in nitrogen with a total pressure of $1 \mathrm{~atm}$.

Figure 13. Comparisons between band absorbtances of pure methane with $l=7.62 \mathrm{~cm}$ and $p=1.013$ atm calculated from the NB database, the NIST database, the EM2C database, HITRAN 2012 and the data of Lee and Happel [36].

Figure 14. Comparison between Planck-mean absorption coefficients for methane calculated from the NB database, the NIST database, the EM2C database, HITRAN 2012 and the correlation of Tien [37]. 
Figure 15. $a_{1}$ ) and $b_{1}$ ) axial profiles of temperature and methane molar concentration for the $34 \mathrm{~kW}$ and the $176 \mathrm{~kW}$ pool fires, respectively. $\mathrm{a}_{2}$ ) et $\mathrm{b}_{2}$ ) axial profiles of radiative intensity for the $34 \mathrm{~kW}$ and the $176 \mathrm{~kW}$ pool fires, respectively. 
$\left(a_{1}\right)$

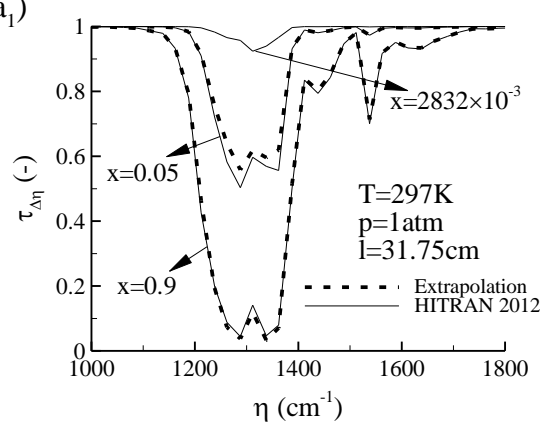

(b)

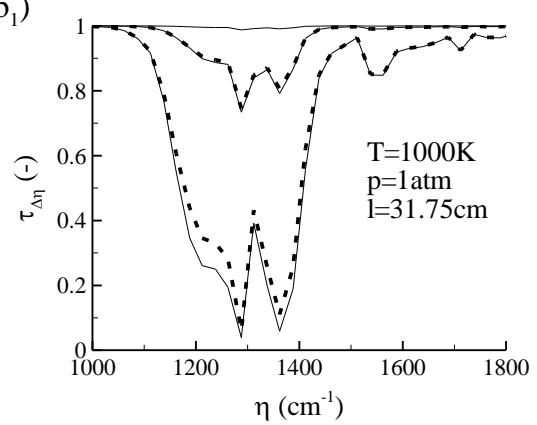

$\left(a_{2}\right)$

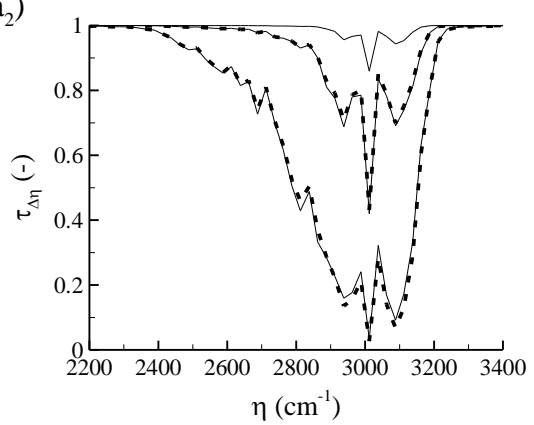

$\left(b_{2}\right)$

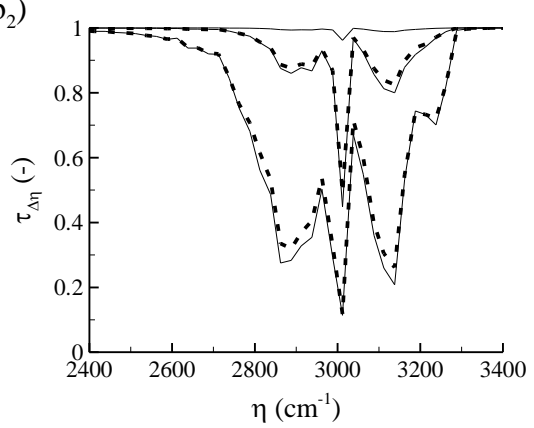

Figure 1. 
$\left(a_{1}\right)$

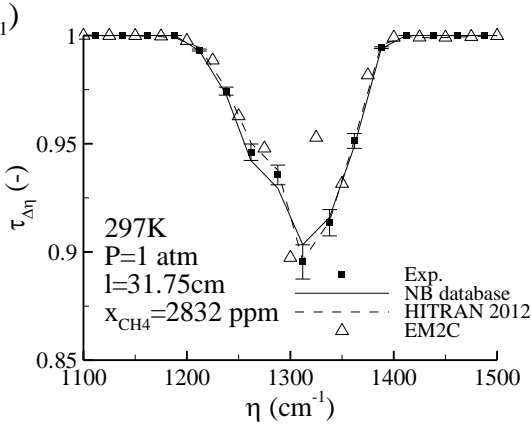

$\left(b_{1}\right)$

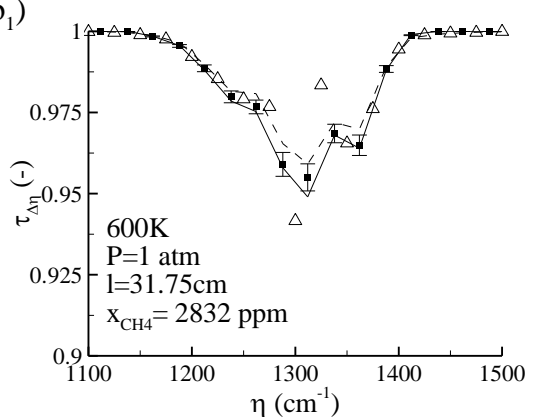

$\left(c_{1}\right)$

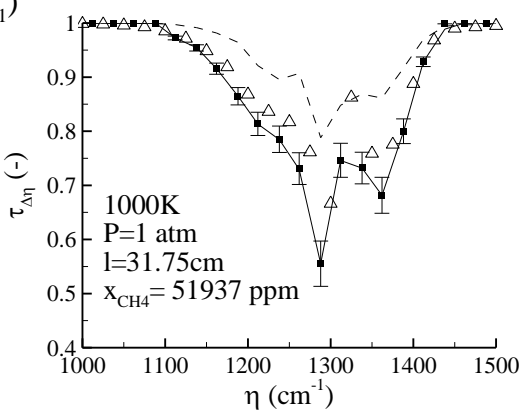

$\left(a_{2}\right)$

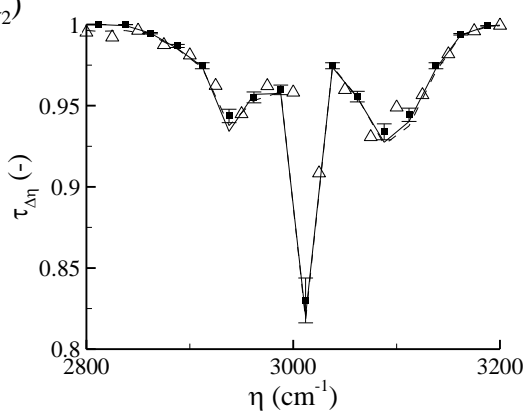

$\left(b_{2}\right)$

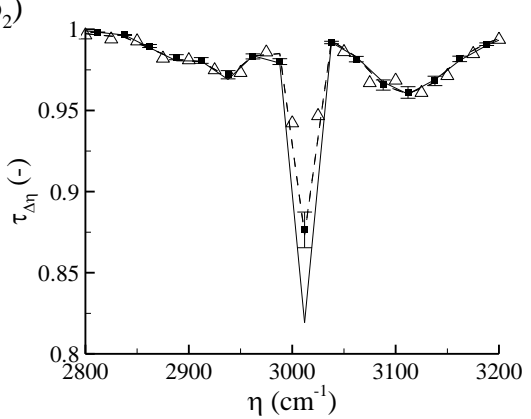

$\left(c_{2}\right)$

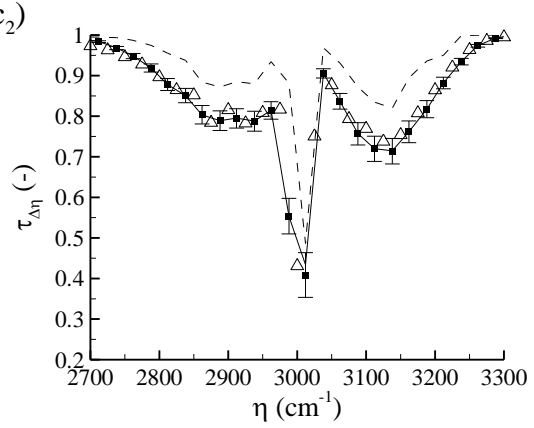

Figure 2. 
$\left(a_{1}\right)$

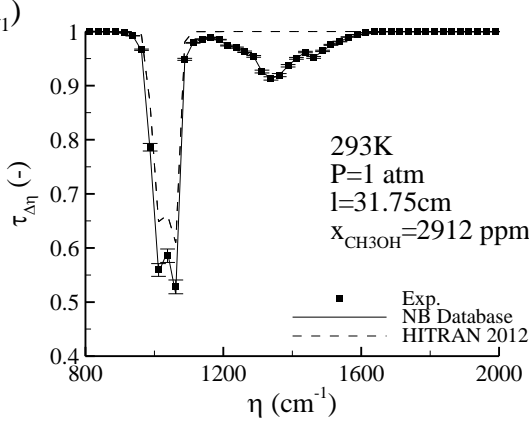

$\left(b_{1}\right)$

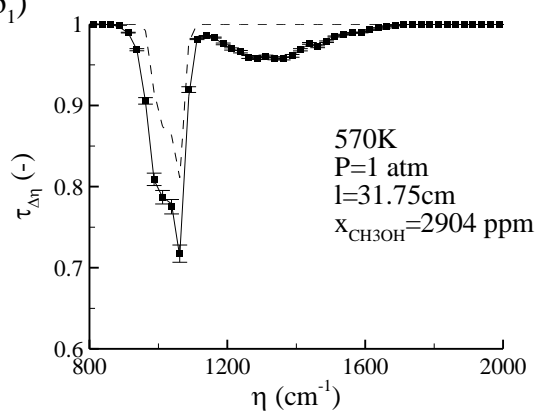

$\left(c_{1}\right)$

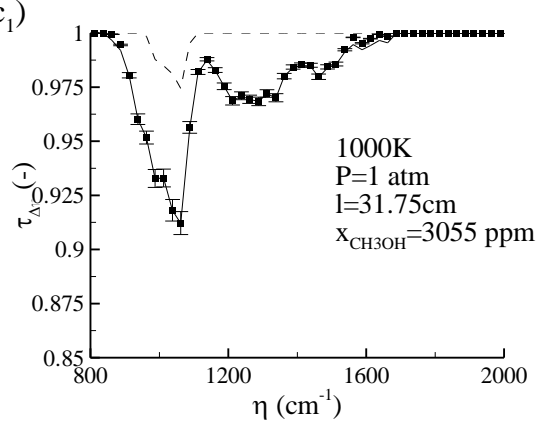

$\left(\mathrm{a}_{2}\right)$

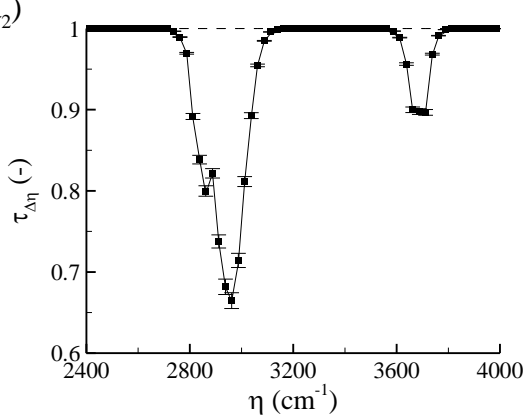

$\left(b_{2}\right)$

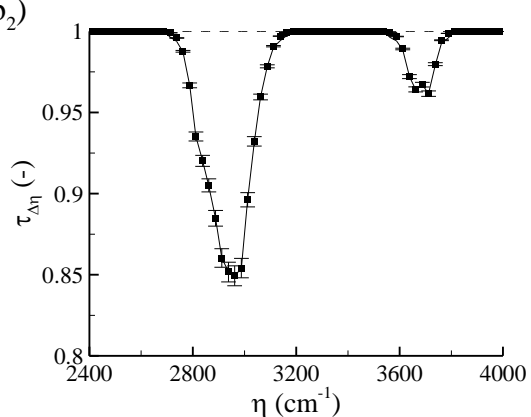

$\left(c_{2}\right)$

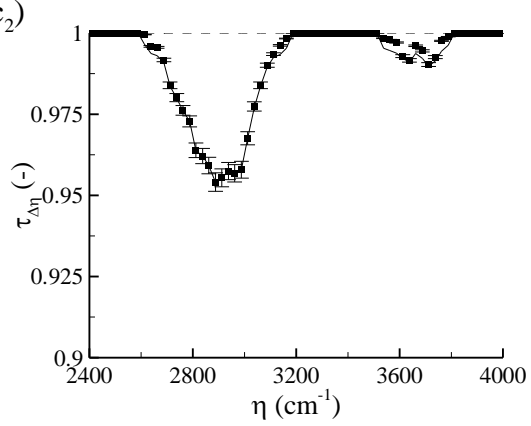

Figure 3. 
$\left(a_{1}\right)$

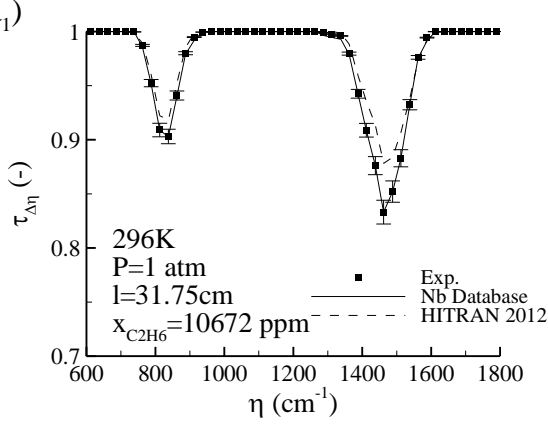

$\left(b_{1}\right)$

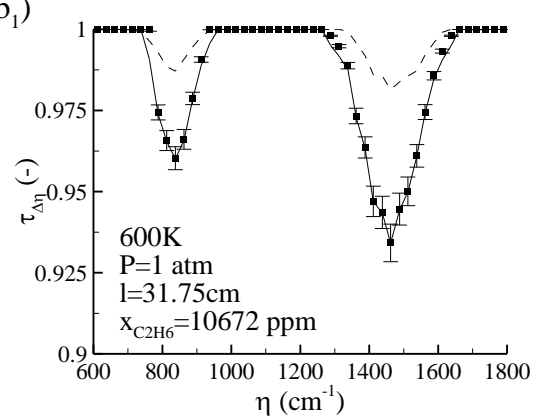

$\left(c_{1}\right)$

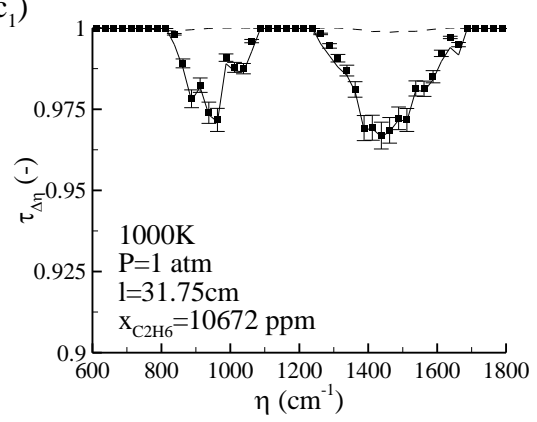

$\left(a_{2}\right)$

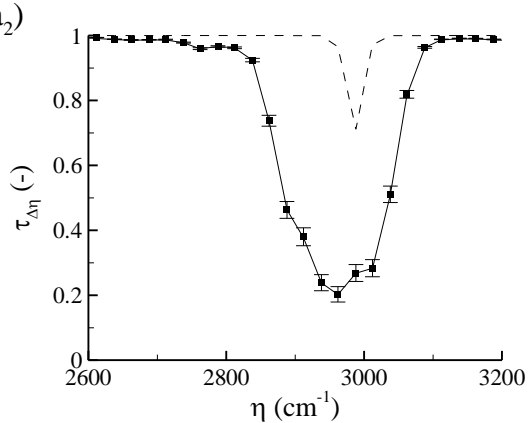

$\left(b_{2}\right)$

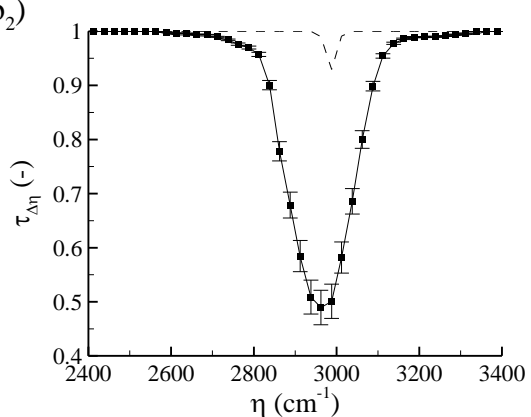

$\left(c_{2}\right)$

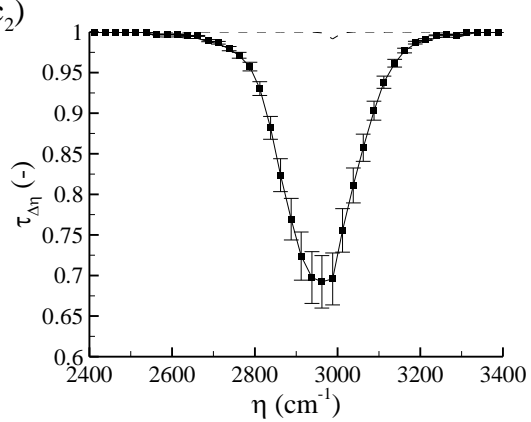

Figure 4. 
$\left(a_{1}\right)$

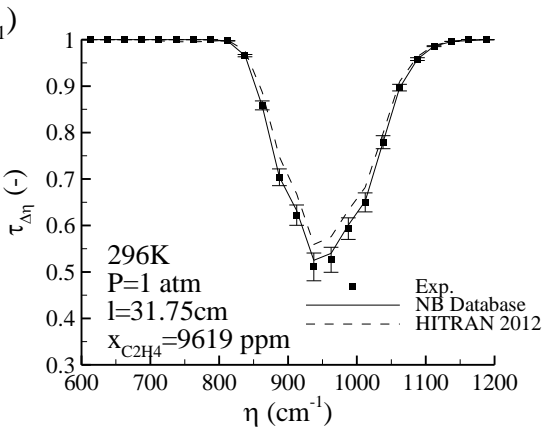

$\left(b_{1}\right)$

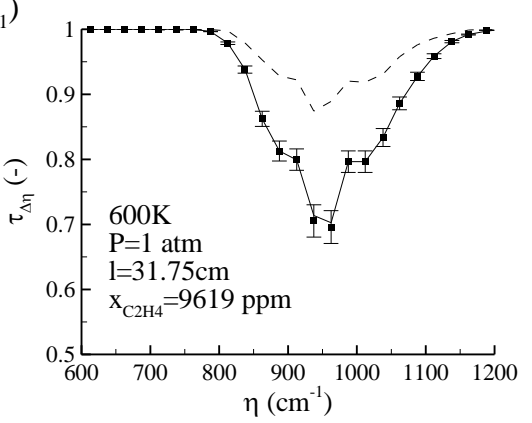

$\left(c_{1}\right)$

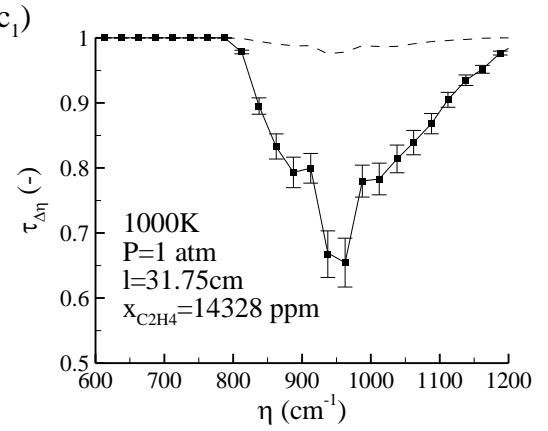

$\left(a_{2}\right)$

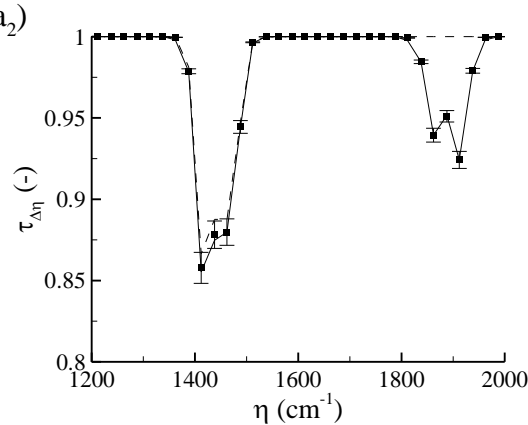

(b)

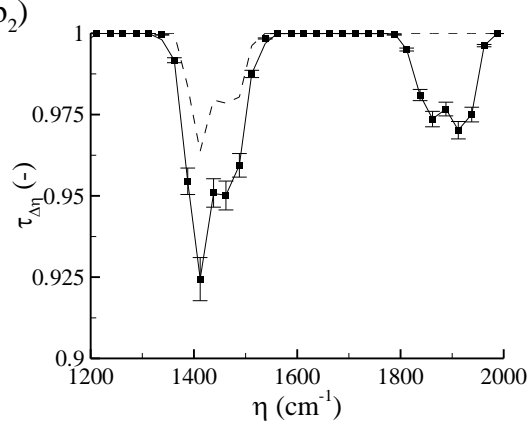

$\left(c_{2}\right)$

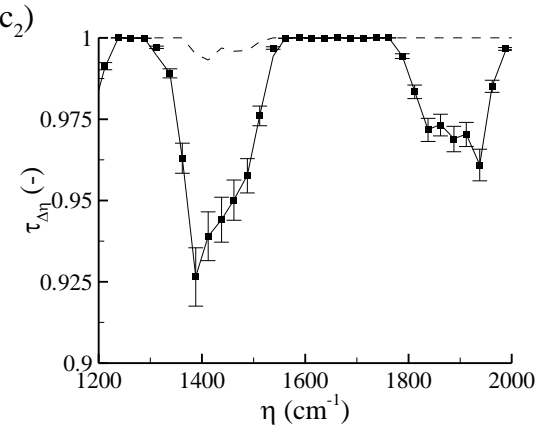

$\left(a_{3}\right)$

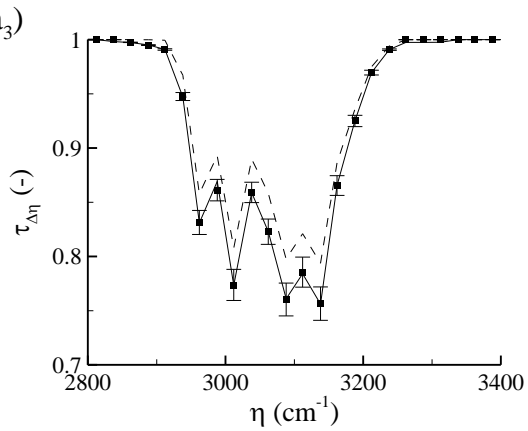

$\left(b_{3}\right)$

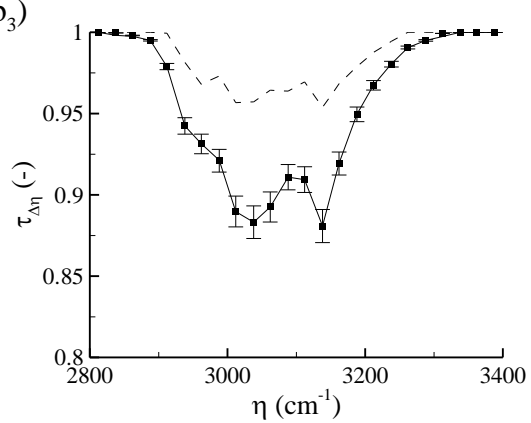

$\left(c_{3}\right)$

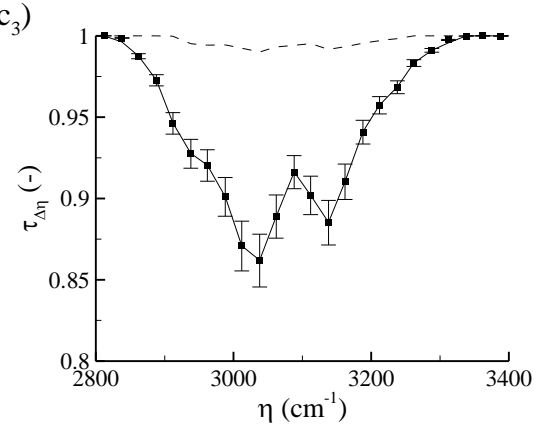

Figure 5. 
$\left(a_{1}\right)$

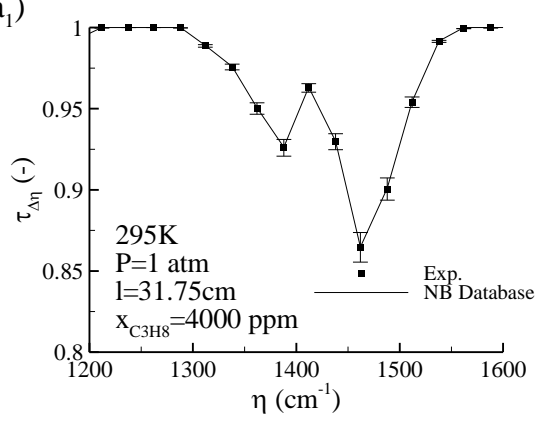

$\left(b_{1}\right)$

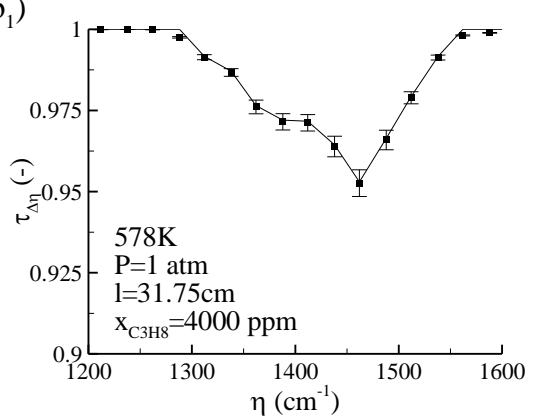

(c $c_{1}$

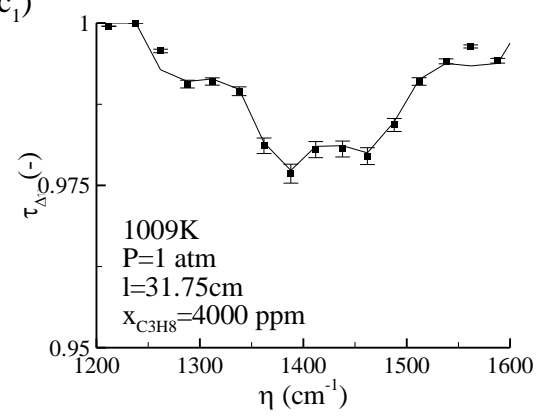

$\left(a_{2}\right)$

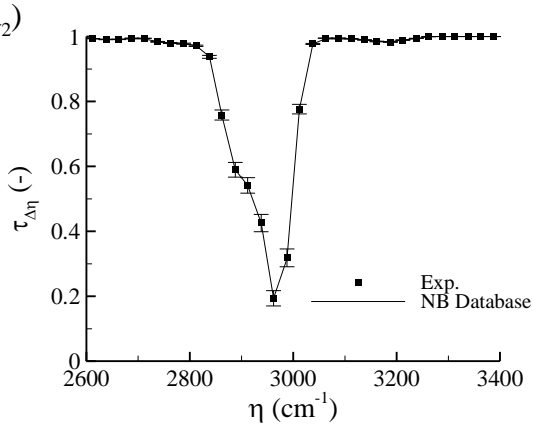

(b)

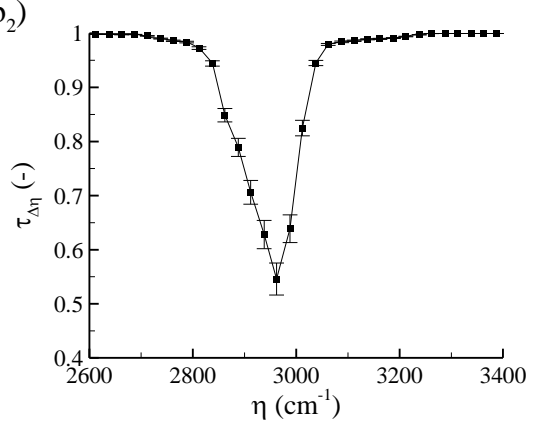

$\left(c_{2}\right)$

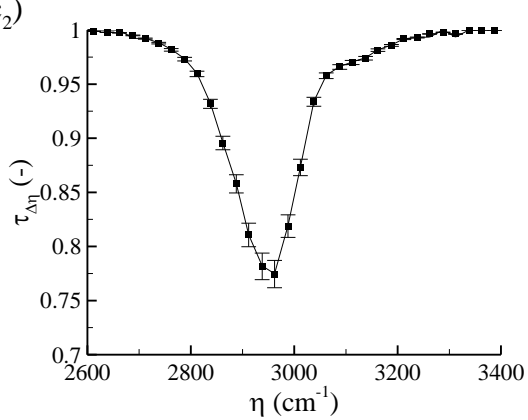

Figure 6. 
$\left(a_{1}\right)$

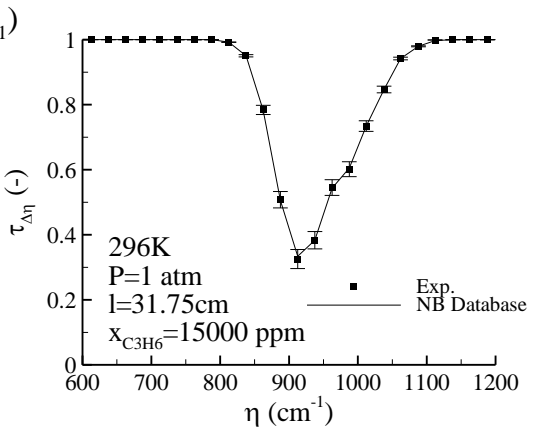

$\left(b_{1}\right)$

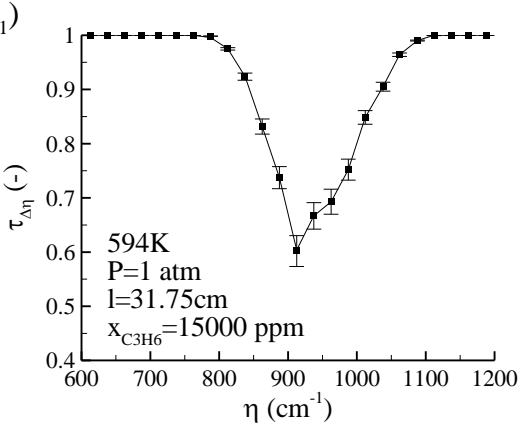

$\left(c_{1}\right)$

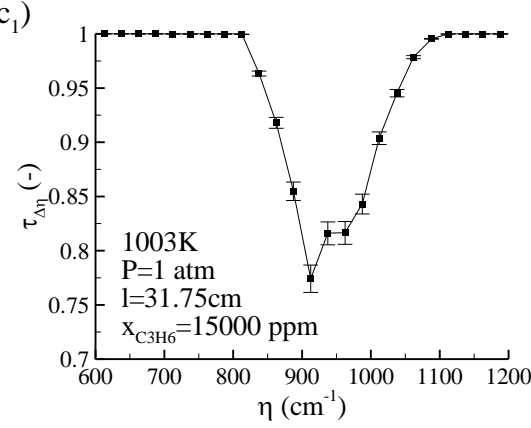

$\left(a_{2}\right)$

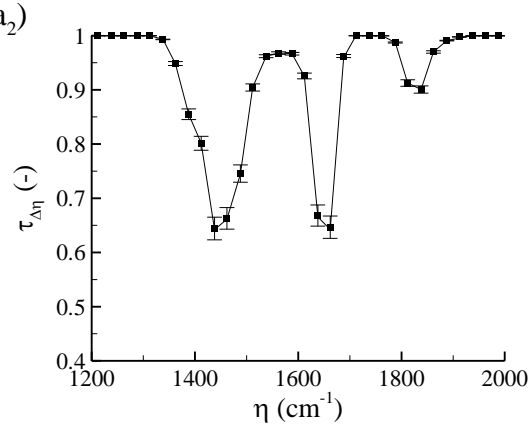

$\left(b_{2}\right)$

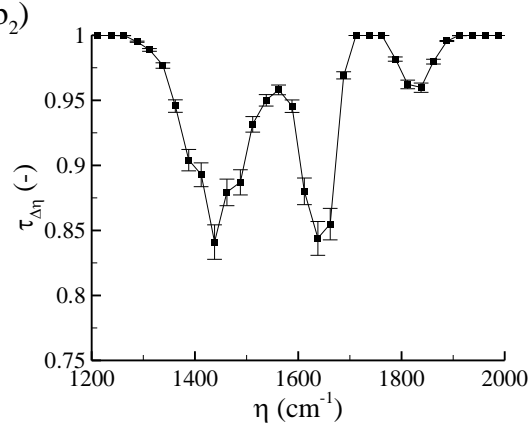

$\left(c_{2}\right)$

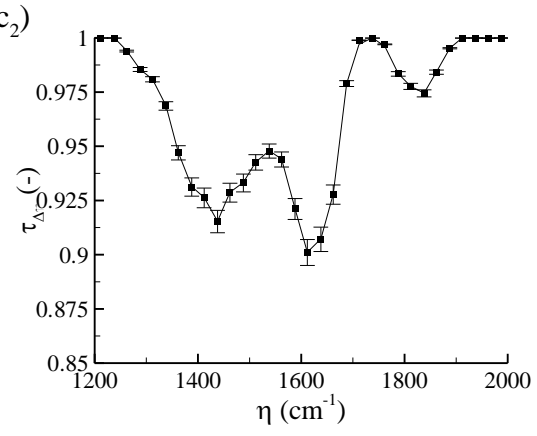

$\left(a_{3}\right)$

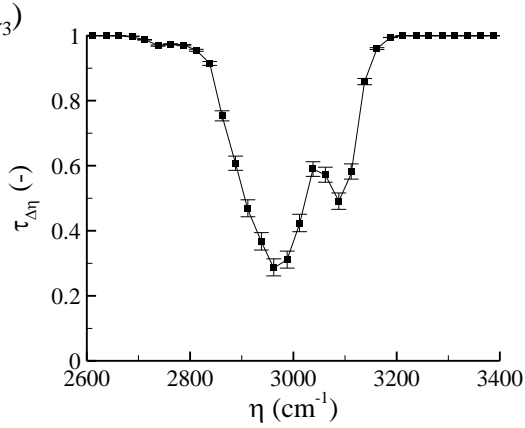

$\left(b_{3}\right)$

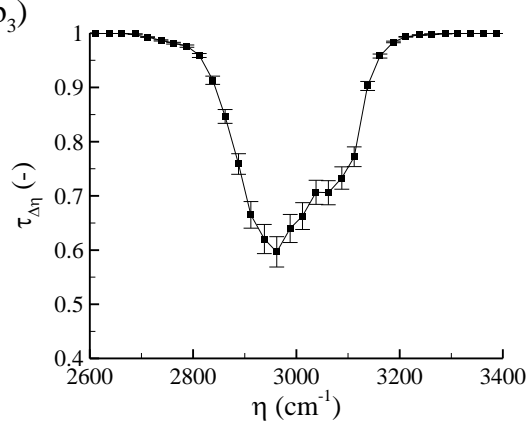

$\left(c_{3}\right)$

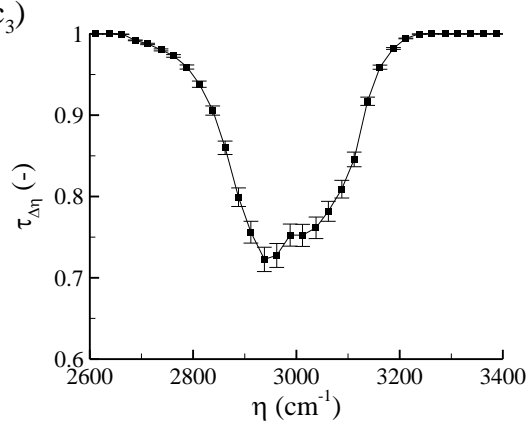

Figure 7. 
$\left(a_{1}\right)$

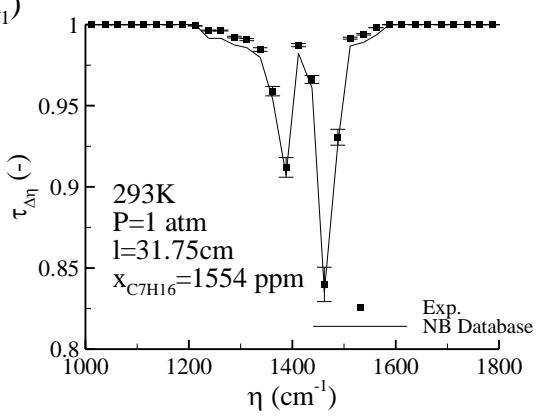

$\left(b_{1}\right)$

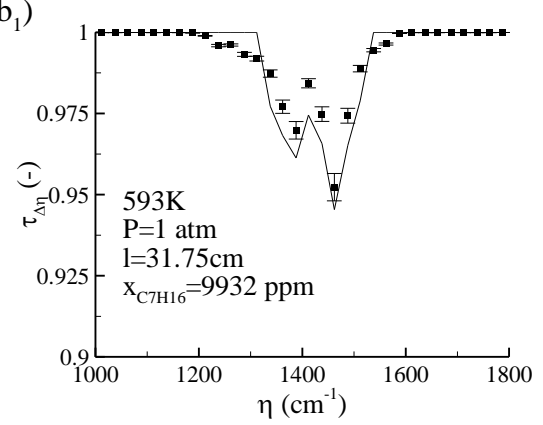

$\left(c_{1}\right)$

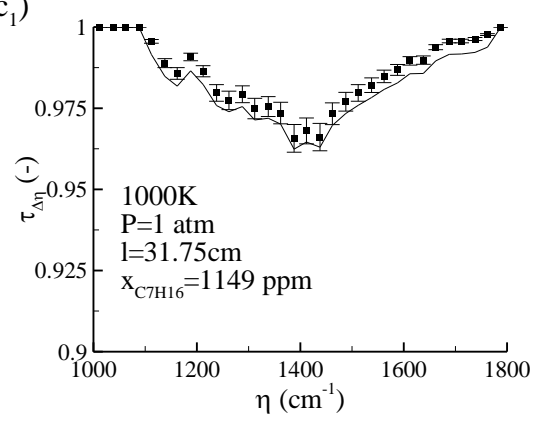

$\left(a_{2}\right)$

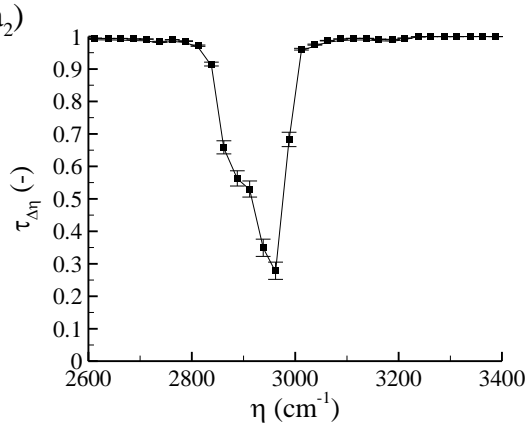

$\left(b_{2}\right)$

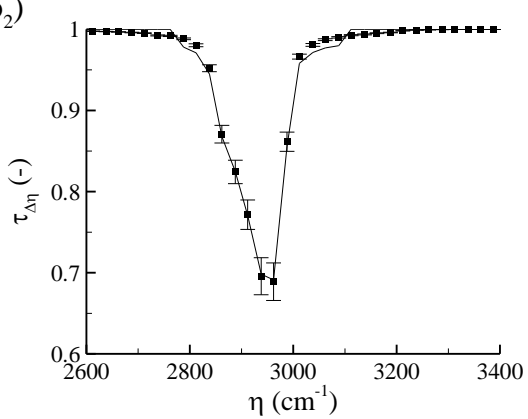

$\left(c_{2}\right)$

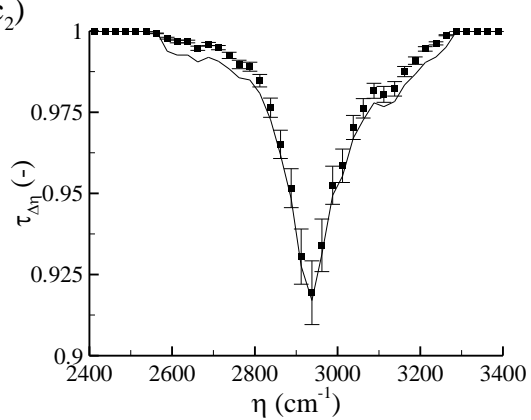

Figure 8. 
$\left(a_{1}\right)$

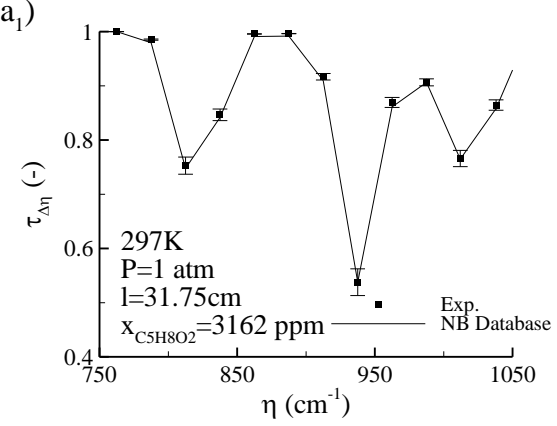

$\left(b_{1}\right)$

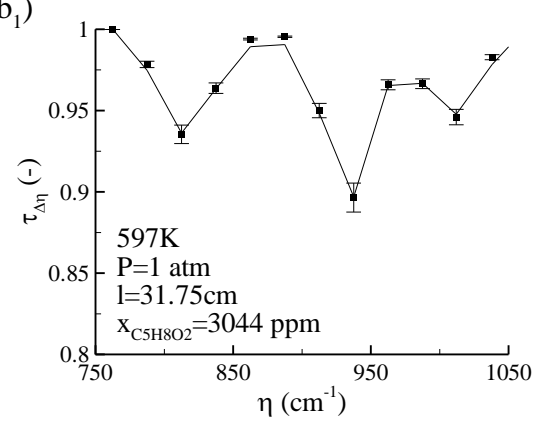

$\left(c_{1}\right)$

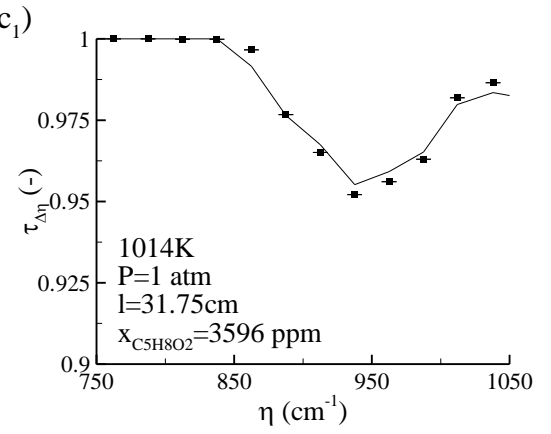

$\left(a_{2}\right)$

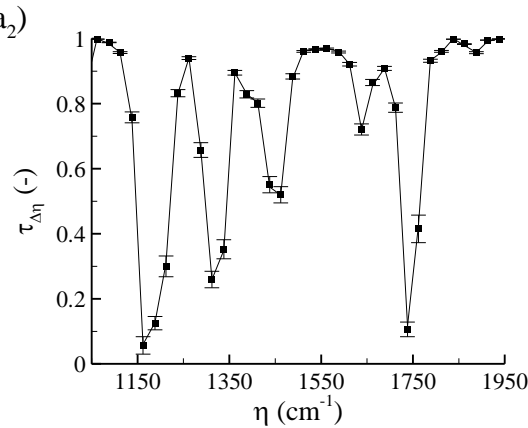

$\left(b_{2}\right)$

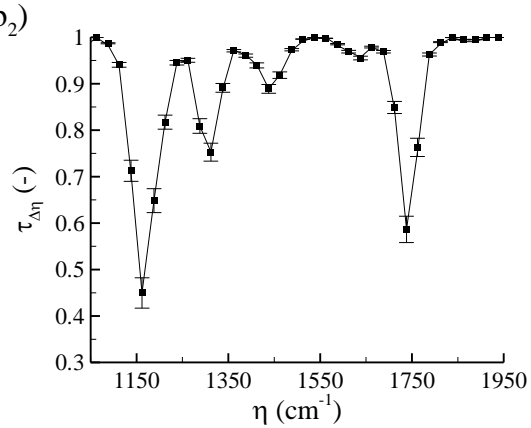

$\left(c_{2}\right)$

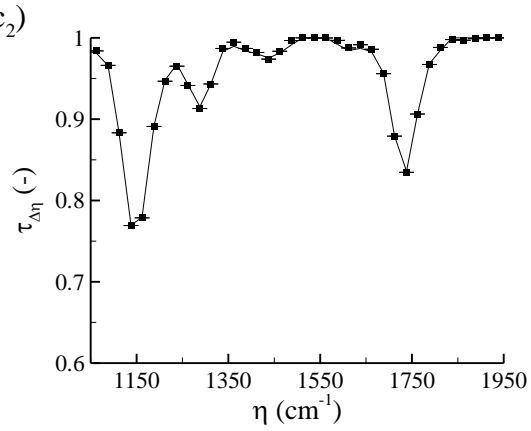

$\left(a_{3}\right)$

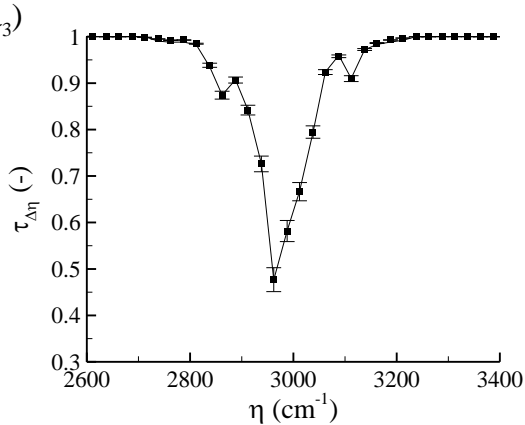

$\left(b_{3}\right)$

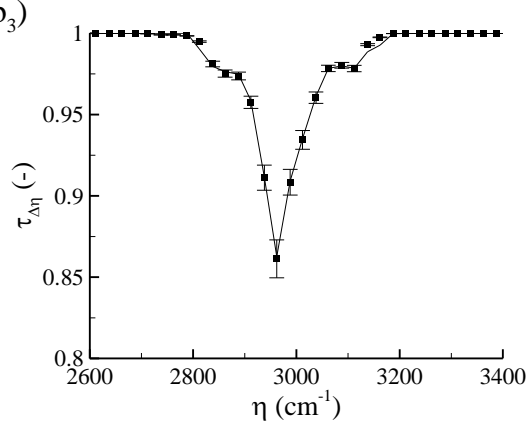

$\left(c_{3}\right)$

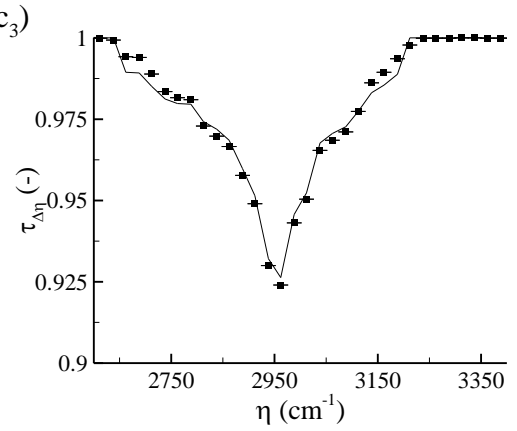

Figure 9. 
$\left(a_{1}\right)$

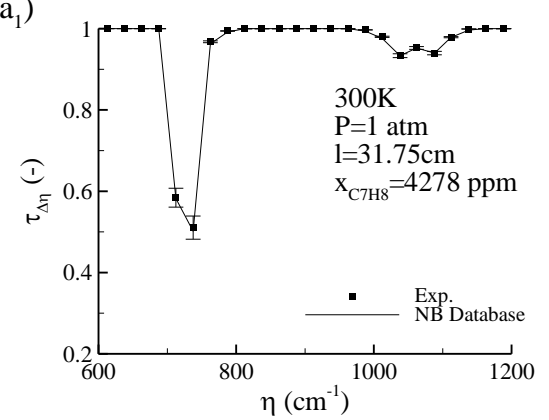

$\left(b_{1}\right)$

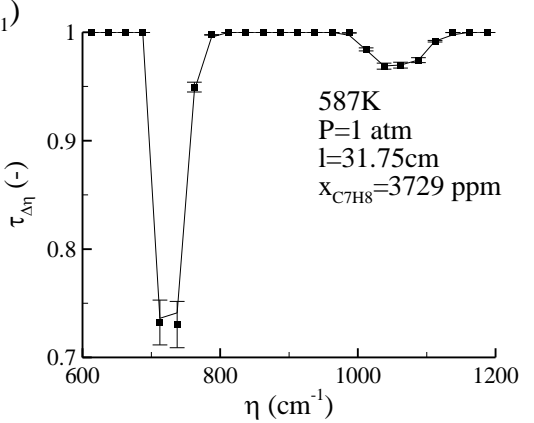

$\left(c_{1}\right)$

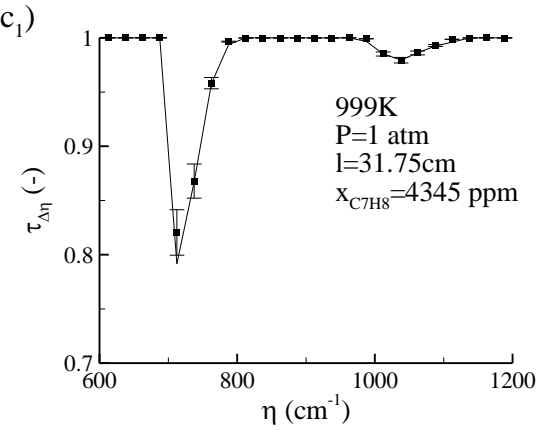

$\left(a_{2}\right)$

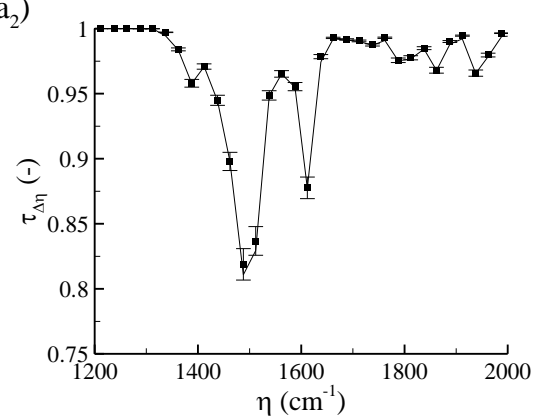

$\left(b_{2}\right)$

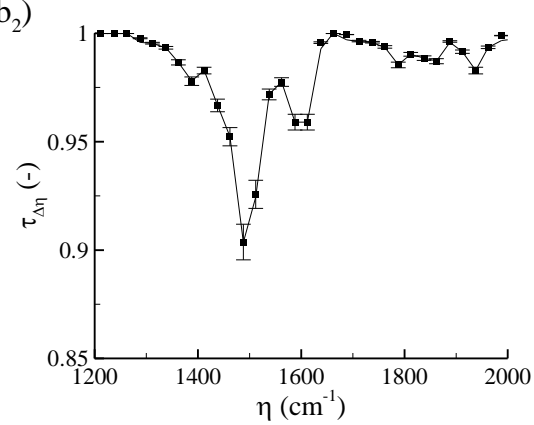

$\left(c_{2}\right)$

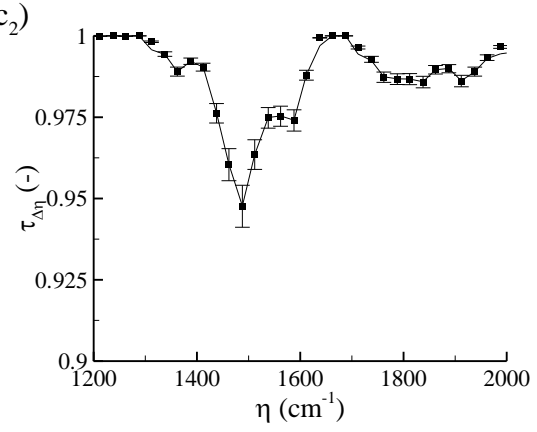

$\left(a_{3}\right)$

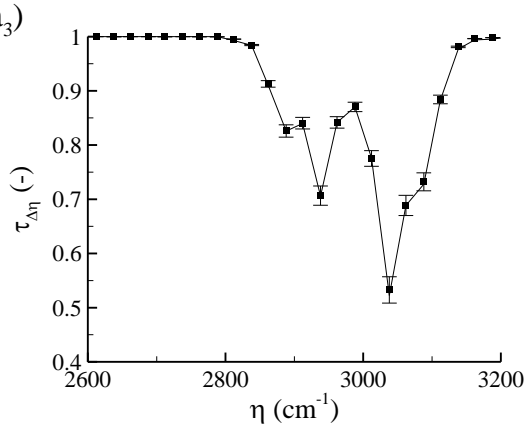

$\left(b_{3}\right)$

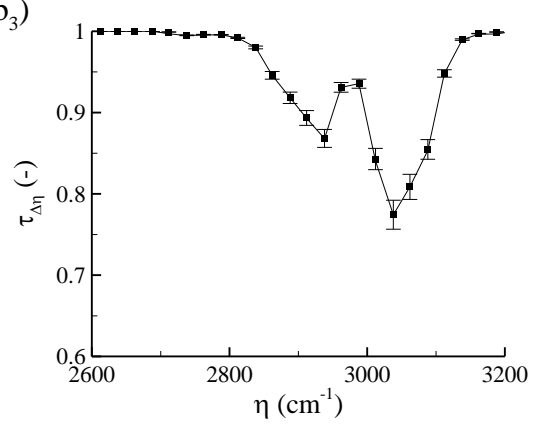

$\left(c_{3}\right)$

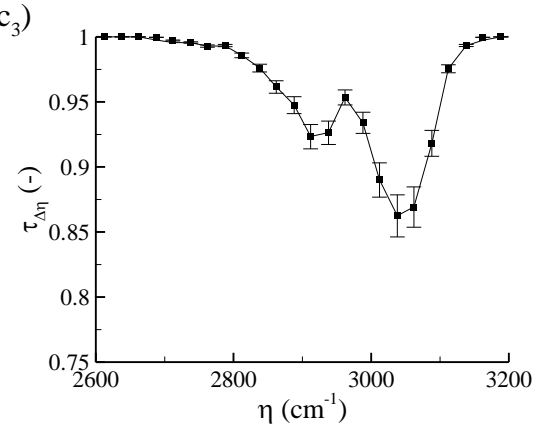

Figure 10. 
$\left(a_{1}\right)$

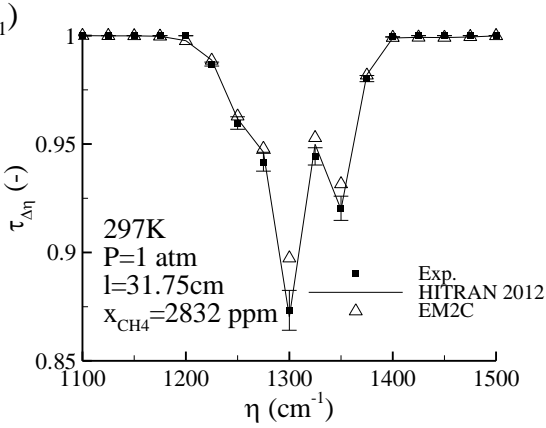

$\left(b_{1}\right)$

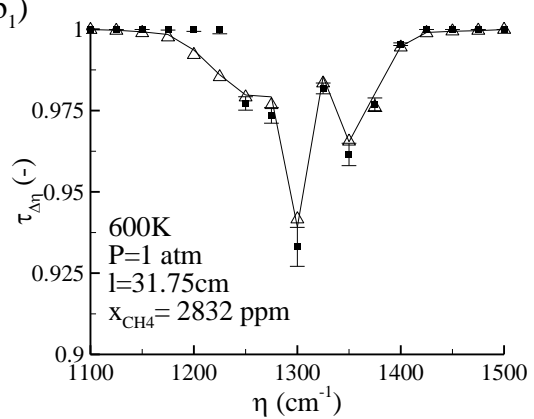

(c)

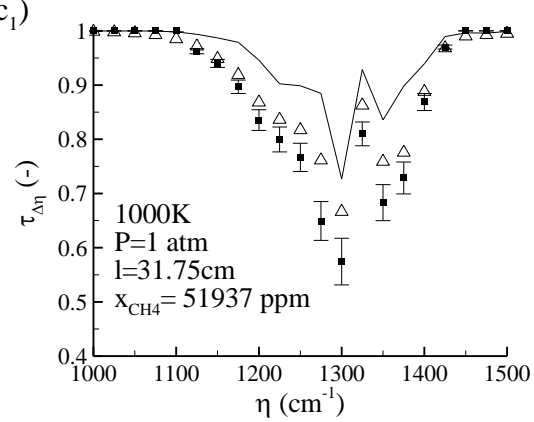

$\left(a_{2}\right)$

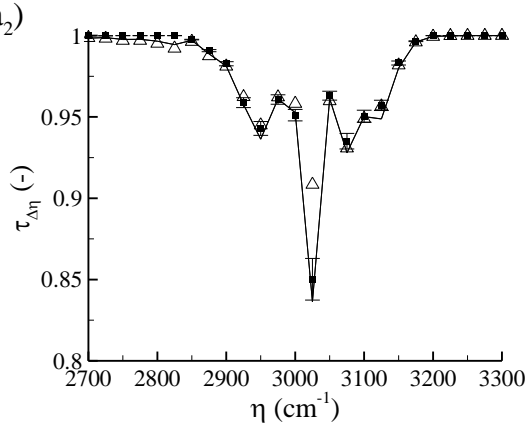

$\left(b_{2}\right)$

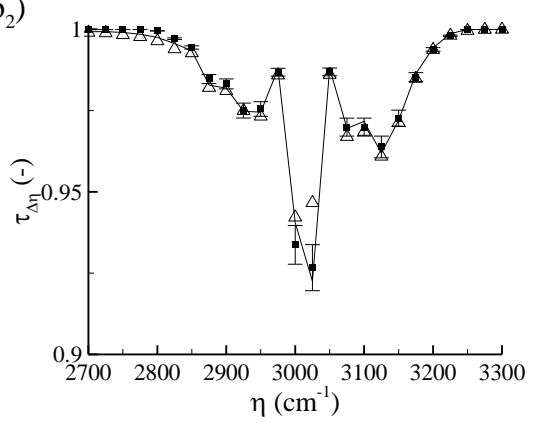

$\left(c_{2}\right)$

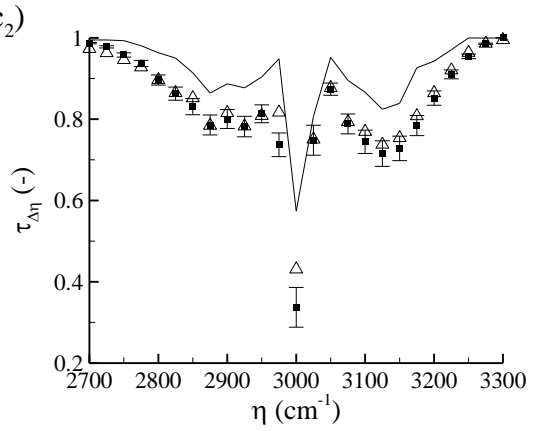

Figure 11. 


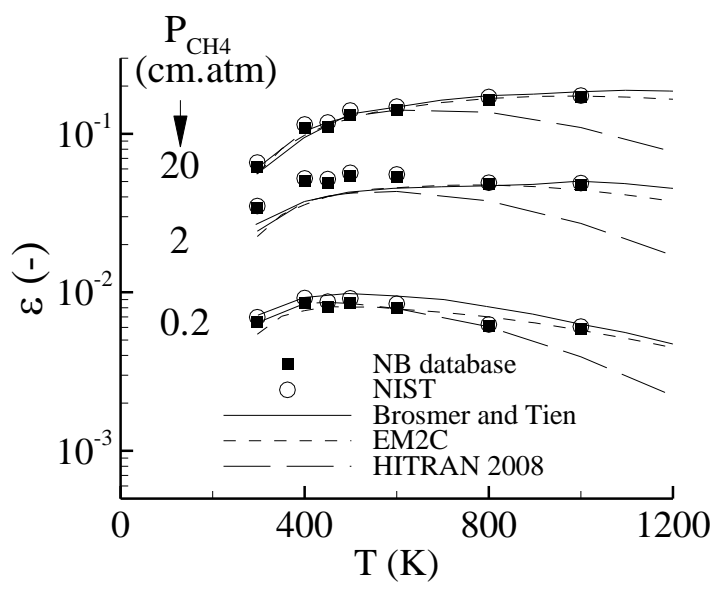

Figure 12. 


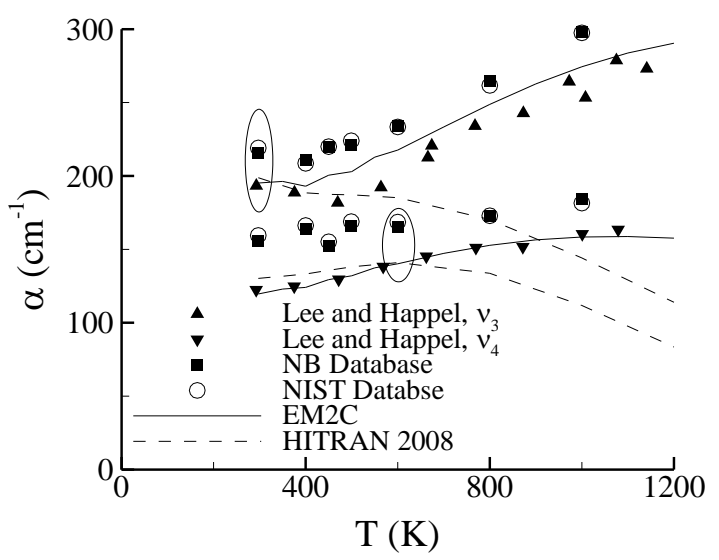

Figure 13. 


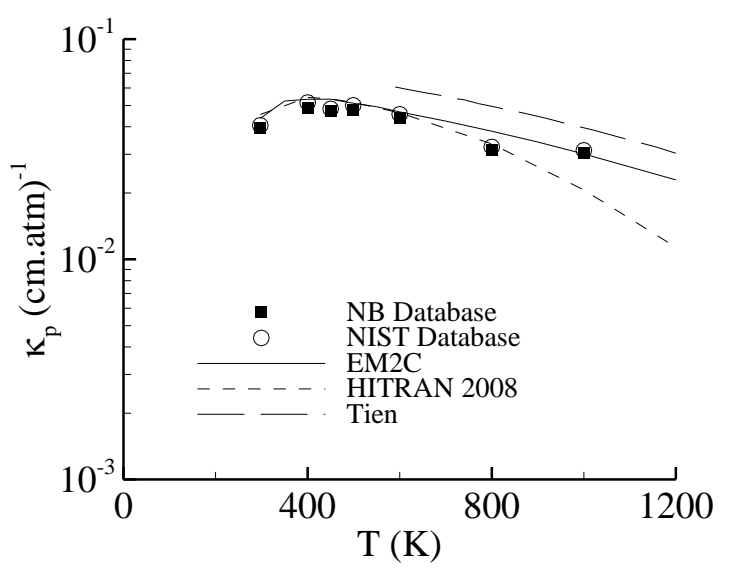

Figure 14. 

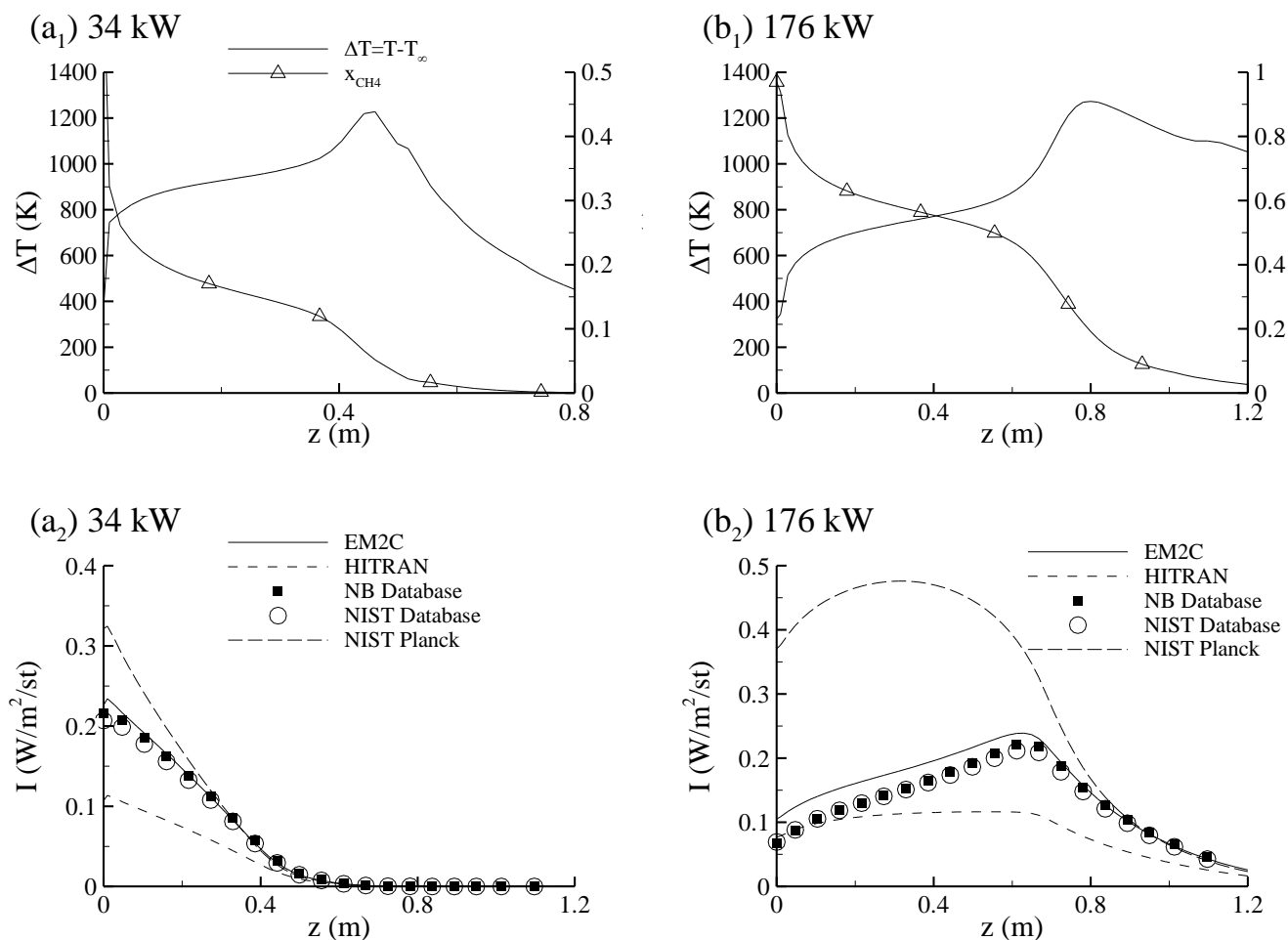

Figure 15. 